\title{
Checklist of hornworts and liverworts of Fiji
}

\author{
Lars Söderström¹, Anders Hagborg², Tamás Pócs ${ }^{3}$, Andrea Sass- \\ Gyarmati ${ }^{3}$, Elizabeth Brown ${ }^{4}$, Matt von Konrat ${ }^{2}$ and Matt Renner ${ }^{4}$
}

${ }^{1}$ Department of Biology, Norwegian University of Science and Technology, N-7491 Trondheim, Norway

${ }^{2}$ Botany Department, The Field Museum, Chicago, Illinois 60605-2496, U.S.A.

${ }^{3}$ Botany Department, Eszterházy College, Eger. Pf. 43, H-3301, Hungary

${ }^{4}$ National Herbarium of New South Wales, Royal Botanic Gardens Sydney, Mrs Macquaries Rd, Sydney NSW 2000, Australia

\begin{abstract}
The first checklist of liverworts and hornworts is provided for the Republic of Fiji. Geographic coverage includes the islands Viti Levu, Vanua Levu, Taveuni, Kadavu, and Ovalau and surrounding smaller islands. There remain many localities in Fiji, including entire islands, which have never or scarcely been collected for this group of plants. We report 11 hornwort taxa and 280 liverwort taxa. An additional 34 taxa previously reported from Fiji are either rejected or noted as doubtful records. The list is based on a compilation of over 250 literature references, including monographs, broader regional studies and molecular investigations. Despite its apparent diversity, the liverwort and hornwort flora of Fiji has received negligible attention, and few publications exist that have focused solely on this group of islands. Knowledge of the liverwort and hornwort flora, of Fiji and regionally for the islands of the South Pacific, remains too poor to reliably comment about range-restricted or endemic species. New records will undoubtedly be reported, of regionally widespread species as well as species likely new to science. A number of the taxa presented here may be synonyms of other taxa as they are poorly known taxonomically. The combination, Frullania parhamii (R.M.Schust.) R.M.Schust. ex von Konrat, L.Söderstr. et A.Hagborg, is made replacing a previously invalid combination.
\end{abstract}

\section{Introduction}

Conservation International identified all the islands of Micronesia and Polynesia, including the islands of Fiji, as the Polynesia-Micronesia hotspot, one of thirty-five biodiversity hotspots in the world (Mittermeier et al. 2005). Alarmingly, Conservation International recognised this hotspot as having one of the highest extinction rates in the world. In Fiji, many localities, including entire islands, currently have never or scarcely been collected for many plant groups (DoE 1997). von Konrat et al. (2011, this issue) provide an overview of the current state of floristic knowledge for Fijian liverworts and hornworts, as well as for lichenised fungi, mosses, ferns and lycophytes for the island group. Historically, very few publications have focused solely on collections from Fiji. 
Prodromus florae hepaticarum polynesiae (Miller et al. 1983) remains a significant work that included Fiji, yet was largely an uncritical compilation of names and literature. We here present the first ever checklist of liverworts and hornworts for the islands of Fiji in an effort to further promote bryological research in the region, and provide a foundation for revisionary studies and floristic research. The checklist presented here provides the critical distinction between those names recorded in the literature as first hand reports where authors have cited specimens and those names that are apparently not based on cited specimens. This checklist fills a major gap in our knowledge of Fijian terrestrial plants. The significance of checklists is summarised by Söderström et al. (2007, 2008), including outlining the utility of checklists as powerful and important tools, and their applicability to taxonomy, systematics, and conservation. Undoubtedly, many new records will be reported for the Fijian islands. This is supported by the fact that at least 75 other liverwort and hornwort species occur on the nearby islands of Samoa alone as well as the report of Pócs et al. (2011, this issue) of 48 new taxa for the islands. In this checklist we report 291 taxa in 286 species as occurring on the Fijian islands. An additional 27 taxa are rejected from the flora of the islands and 7 are reported as doubtful records.

\section{Methodology and materials}

In this checklist the taxa are arranged in alphabetic order. The list is in three parts, i) accepted reports, ii) doubtful, iii) excluded reports. Finally, for ease of reference, a list of all synonyms applied in the checklist or names of alternative use is given.

Each accepted taxon is qualified using a four level ranking system that indicates our level of confidence about a taxon's value. The coding convention is outlined in detail by von Konrat et al. (2010a) and the following provides a brief explanation:

? Problem with the taxon name and value. The taxon is invalid, illegitimate or 'orphaned' in a synonymised genus or species but its correct placement is unknown.

* Serious doubts. There are doubts about the value of the taxon. It can be that there are conflicting views without any substantial evidence in any direction, conflicting views with substantiating evidence supporting one or both positions, or there is evidence pointing towards synonymization but it is premature to do it.

** Knowledge problem. The taxon is not well known by the person evaluating it. It may be a newly described species or a species originally not well described and not restudied recently.

${ }^{* * *}$ Accepted. A good taxon as currently understood based on personal experience or on a convincing taxonomic revision. Nomenclature and/or taxonomic position may, however, be questioned, e.g. elements may be excluded from the taxon, but the taxon with the current type will still be accepted.

Additional notes are provided throughout explaining the scoring for individual taxa, especially for taxa that that are denoted ${ }^{*}$.

This checklist provides a critical distinction between names recorded in the literature as first hand reports, i.e. "A report citing a specimen or an observation of material either in the field 'and then locality fairly exact' or among collected specimens" (bold font) and second hand reports, i.e. "Merely citing a locality that is not exact enough to 
be searched for the species, and not apparently based on a specimen that can be found" (regular font) (see von Konrat et al. 2010a). This will greatly aid further evaluation of these names for Fiji. Moreover, the list includes all types of Fijian origin.

In order to condense the list we use the words "both as" or "all as" to denote that the author used this name(s) in both/all publications by the same author. The word "also" indicates that the author(s) used the current accepted name in addition to another name.

Island abbreviations are as follows: Viti Levu (VI), Vanua Levu (VA), Taveuni (TA), Kadavu (KA), Ovalau (OV, including nearby smaller islands) and Lau group (LA). We make an attempt to order the reports by these provinces. The abbreviation FIJ is used for reports that are not specific enough to be allocated to an island.

\section{Nomenclatural novelty}

Frullania parhamii (R.M.Schust.) R.M.Schust. ex von Konrat, L.Söderstr. et A.Hagborg, comb. nov.

Basionym: Neohattoria parhamii R.M.Schust. J. Hatt. Bot. Lab. 26: 243, 1963. Type: Summit of Mt Delaikoro (Ndelaikoro), south of Lambasa, Vanua Levu, Fiji, on bark in open rain forest, R. M. Schuster 56709, June 1962 (holotype, F).

Synonym: Frullania parhamii (R.M.Schust.) R.M.Schust. Hepat. Anthocerotae N. Amer. 5: 34, 1992, nom. inval. (Art. 33.4; basionym not cited; McNeill et al. 2006).

\section{Checklist of taxa accepted from Fiji}

Current list includes all taxa that we accept as occurring in Fiji. However, the taxonomic value of some of them is questionable.

\section{Anthocerotophyta}

\section{Dendroceros}

*** D. cavernosus J.Haseg. FIJ: Hasegawa 1986b, 2002. VI: Hasegawa 1986a.

* $\quad{ }^{1}$ D. crispatus (Hook.) Nees VI: Campbell 1971, Miller et al. 1983.

*** D. granulatus Mitt. VI: Hasegawa 1986a.

*** D. javanicus (Nees) Nees FII: Hasegawa 1986b. VI: Gibbs 1909, Hürlimann 1960 as Dendroceros pusillus, Miller et al. 1983 also as Dendroceros pusillus, Hasegawa 1986a.

\section{Folioceros}

*** F. amboinensis (Schiffn.) Piippo FIJ: Hasegawa 1993 as Anthoceros amboinensis, Piippo 1993. VI: Hasegawa 1986a as Folioceros spinisporus.

${ }^{1}$ Dendroceros crispatus is treated as a synonym of Dendroceros crispus (Sw.) Nees by Gradstein \& Costa (2003) but accepted as a species by J.C.Villarreal (pers. comm.). 
*** F. fuciformis (Mont.) D.C.Bharadwaj VI: Campbell 1971 as Anthoceros fuciformis, Miller et al. 1983 as Anthoceros fuciformis.

*** F. glandulosus (Lehm. et Lindenb.) D.C.Bharadwaj FIJ: Schiffner 1898 as Anthoceros glandulosus, Schiffner 1955 as Aspiromitus glandulosus, Hasegawa 2002 as Anthoceros appendiculatus. VI: Schiffner 1890 as Anthoceros glandulosus, Miller et al. 1983 as Anthoceros glandulosus.

*** F. pinnilobus (Steph.) D.C.Bharadwaj VI: Hasegawa 1986a.

\section{Hattorioceros}

** H. striatisporus (J.Haseg.) J.Haseg. VI: Hasegawa 2000.

\section{Megaceros}

*** M. flagellaris (Mitt.) Steph. FIJ: Stephani 1916, Miller et al. 1983 as Anthoceros flagellaris. VI: Gibbs 1909 as Anthoceros flagellaris, Hürlimann 1960 as Megaceros monospirus, Miller et al. 1983 also as Megaceros monospirus Steph., Hasegawa 1986a.

\section{Phaeoceros}

*** P. carolinianus (Michx.) Prosk. FII: Hasegawa 1984 as Phaeoceros laevis subsp. carolinianus, Schuster 1992b as Anthoceros laevis subsp. carolinianus, Piippo 1993 as Phaeoceros laevis subsp. carolinianus, Guo \& Zhang 1999 as Phaeoceros laevis subsp. carolinianus, Damsholt 2002 as Phaeoceros laevis subsp. carolinianus. VI: Campbell 1971 as Phaeoceros laevis subsp. carolinianus, Miller et al. 1983 as Phaeoceros laevis subsp. carolinianus.

\section{Marchantiophyta}

\section{Acrolejeunea}

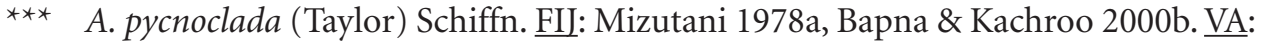
Campbell 1971 as Ptychocoleus pycnocladus, Miller et al. 1983. VI: Miller et al. 1983.

*** - subsp. pycnoclada (Taylor) Schiffn. KA: Pócs et al. 2011. VI: Gradstein 1975, Hürlimann 1989.

\section{Aneura}

*** A. maxima (Schiffn.) Steph. VA: Campbell 1971 as Riccardia maxima, Miller et al. 1983 as Riccardia maxima. VI: Gibbs 1909, Miller et al. 1983 as Riccardia maxima.

\section{Archilejeunea}

* $\quad{ }^{2}$ A. bilabiata (Mitt.) Steph. FII: TYPE of Phragmicoma bilabiata, Mitten 1873 as Phragmicoma bilabiata, Stephani 1911, Bonner 1962b, Thiers 1983 as Phragmicoma bilabiata, Miller et al. 1983 also as Phragmicoma bilabiata, Geissler \& Bischler 1989 as Phragmicoma bilabiata.

\footnotetext{
${ }^{2}$ Archilejeunea bilabiata is a doubtful species (type unknown) (Geissler \& Gradstein 1994).
} 
** A. brachyantha J.B.Jack et Steph. FII: Stephani 1911, Verdoorn 1934. OV: TYPE, Jack \& Stephani 1894, Bonner 1962b, Campbell 1971, Miller et al. 1983. VA: Campbell 1971, Miller et al. 1983. VI: Campbell 1971, Miller et al. 1983.

*** A. planiuscula (Mitt.) Steph. KA: Pócs et al. 2011. TA: Pócs et al. 2011. $\underline{\text { VA: }}$ Campbell 1971 as Archilejeunea mariana, Miller et al. 1983 as Spruceanthus marianus. VI: Campbell 1971 as Archilejeunea mariana, Miller et al. 1983 as Spruceanthus marianus.

\section{Bazzania}

** B. caudistipula (Steph.) Inoue et H.A.Mill. FII: Bonner 1977. VI: TYPE of Mastigobryum caudistipulum, Stephani 1908b as Mastigobryum caudistipulum, Inoue \& Miller 1965, Miller et al. 1983 also as Mastigobryum caudistipulum, Meagher 2010.

** B. combinata (J.B.Jack et Steph.) Steph. VI: Gibbs 1909 as Mastigobryum combinatum, Miller et al. 1983.

*** B. erosa (Reinw., Blume et Nees) Trevis. VI: Campbell 1971, Miller et al. 1983.

** B. falcifolia (Steph.) H.A.Mill. TA: Pócs et al. 2011.

** B. hamatifolia (Steph.) H.A.Mill. FII: TYPE of Mastigobryum hamatifolium, Miller et al. 1983 also as Mastigobryum hamatifolium.

** B. insignis (De Not.) Trevis. VI: Hürlimann 1985.

** B. manillana (Gottsche ex Steph.) S.Hatt. TA: Pócs et al. 2011.

** B. paradoxa (Sande Lac.) Steph. FII: Stephani 1897, Evans 1932, Kitagawa 1967, Piippo et al. 2002. VI: Jack \& Stephani 1894 as Mastigobryum paradoxum, Miller et al. 1983 also as Mastigobryum paradoxum.

** B. subacuta (Mitt.) Steph. VI: Hürlimann 1985.

*** B. tridens (Reinw., Blume et Nees) Trevis. FII: Stephani 1908a as Mastigobryum australe, Jovet-Ast 1951 as Bazzania australe, Kitagawa 1978 as Bazzania australis, Geissler \& Bischler 1985 as Mastigobryum australe, Hürlimann 1985 as Bazzania australis. OV: TYPE of Herpetium australe, Montagne 1843 as Herpetium australe, Bonner 1965 as Herpetium australe, Campbell 1971, Miller et al. 1983. TA: Campbell 1971, Miller et al. 1983. VA: Campbell 1971, Miller et al. 1983, Miller et al. 1983 as Bazzania australis, Mastigobryum australe and Herpetium australe. VI: Campbell 1971, Kitagawa 1973a as Bazzania australis, Miller et al. 1983, Hürlimann 1985.

*** B. uncigera (Reinw., Blume et Nees) Trevis. FII: Stephani 1908b as Mastigobryum uncigerum, Kitagawa 1967, 1978, Hattori 1975c, Bapna \& Kachroo 2000. OV: Jack \& Stephani 1894 as Mastigobryum uncigerum, Miller et al. 1983.

** B. vitiana Mitt. ex Steph. FII: TYPE, TYPE of Mastigobryum vitiense, Stephani 1908a as Mastigobryum vitiense, Bonner 1963a, Miller et al. 1983 as Mastigobryum vitiense. OV: Campbell 1971 as Bazzania vitiensis, Miller et al. 1983.VA: Campbell 1971 as Bazzania vitiensis, Miller et al. 1983. VI: Campbell 1971 as Bazzania vitiensis.

*** B. vittata (Gottsche) Trevis. VI: Campbell 1971, Miller et al. 1983, Hürlimann 1985. 


\section{Caudalejeunea}

*** C. reniloba (Gottsche) Steph. FII: Miller et al. 1983 as Lopholejeunea contractilis and Phragmicoma contractilis, Bapna \& Kachroo 2000b, Gradstein et al. 2002. OV: Jack \& Stephani 1894 as Caudalejeunea recurvistipula, Campbell 1971, Miller et al. 1983. TA: Pócs et al. 2011. VA: Campbell 1971, Miller et al. 1983. VI: Campbell 1971, Miller et al. 1983, Mizutani 1988, Hürlimann 1989, Thiers \& Gradstein 1989.

\section{Ceratolejeunea}

*** C. belangeriana (Gottsche) Steph. FII: Hürlimann 1995. KA: Pócs et al. 2011. OV: Campbell 1971, Miller et al. 1983. VI: Campbell 1971, Miller et al. 1983.

*** C. vitiensis Steph. FIJ: TYPE, Stephani 1913, Bonner 1963b. VI: Miller et al. 1983 [Viti Levu with a “?”].

\section{Cheilolejeunea}

** C. ceylanica (Gottsche) R.M.Schust. et Kachroo KA: Pócs et al. 2011. TA: Pócs et al. 2011. VI: Campbell 1971 as Pycnolejeunea ceylanica, Miller et al. 1983, Hürlimann 1995.

** C. cookiensis (Steph.) R.M.Schust. et Kachroo FIJ: Mizutani 1972 as Cheilolejeunea parkinsonii. OV: TYPE of Euosmolejeunea parkinsonii, Stephani 1914 as Euosmolejeunea parkinsonii, Miller 1960 as Euosmolejeunea parkinsonii, Mizutani 1967 as Cheilolejeunea parkinsonii, Miller et al. 1983 also as Euosmolejeunea parkinsonii.

*** C. decursiva (Sande Lac.) R.M.Schust. VI: Zhu \& Lai 2005.

*** C. falsinervis (Sande Lac.) R.M.Schust. et Kachroo FIJ: Thiers 1992a, Thiers 1992b, Yang \& Lin 2008. VI: Campbell 1971 as Pycnolejeunea falsinervis, Miller et al. 1983.

** C. gardneri (Mitt.) Mizut. OV: Campbell 1971 as Pycnolejeunea setifera, Miller et al. 1983 as Pycnolejeunea setifera.

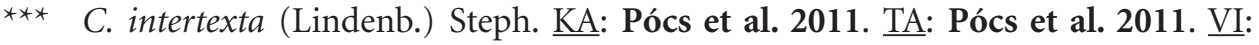
Hürlimann 1995.

*** C. lindenbergii (Gottsche) Mizut. TA: Pócs et al. 2011.

** C. meyeniana (Nees, Lindenb. et Gottsche) R.M.Schust. et Kachroo FII: Thiers 1992a, 1992b, Pócs et al. 1994. OV: Jack \& Stephani 1894 as Archilejeunea meyeniana, Campbell 1971 as Pycnolejeunea meyeniana, Miller et al. 1983 also as Lejeunea meyeniana.

** C. occlusa (Herzog) T.Kodama et N.Kitag. VI: Pócs et al. 2011.

*** C. trapezia (Nees) R.M.Schust. et Kachroo FII: Zhu \& Grolle 2004. KA: Pócs et al. 2011. TA: Pócs et al. 2011. VI: Campbell 1971 as Pycnolejeunea trapezia, Miller et al. 1983.

*** C. trifaria (Reinw., Blume et Nees) Mizut. KA: Pócs et al. 2011. OV: Jack \& Stephani 1894 as Euosmolejeunea partiicola ["paritiicola”], Miller et al. 1983 also as Euosmolejeunea partiicola. VI: Campbell 1971 as Euosmolejeunea trifaria, Miller et al. 1983, Hürlimann 1995. 


\section{Chiastocaulon}

*** C. dendroides (Nees) Carl FIJ: Stephani 1903a as Plagiochila dendroides, JovetAst 1951, Bonner 1963b, Inoue 1984, 1986 both as Plagiochila dendroides, Piippo 1989a as Plagiochila dendroides. VI: Inoue 1970, Hürlimann 1974, Miller et al. 1983.

\section{Chiloscyphus}

** C. graeffeanus Steph. OV: TYPE, Stephani 1922 [“Oahu”], Bonner 1963b, Miller et al. 1983.

*** C. muricatus (Lehm.) J.J.Engel et R.M.Schust. FII: Frye \& Clark 1944 as Lophocolea muricata, Schuster 1980b as Lophocolea muricata. VI: Gibbs 1909 as Lophocolea muricata, Miller et al. 1983 as Lophocolea muricata, Hürlimann 1998 as Lophocolea muricata.

** C. parvus (Steph.) J.J.Engel et R.M.Schust. IIJ: Hürlimann 1998 as Lophocolea parva. VA: Campbell 1971 as Lophocolea parva, Miller et al. 1983 as Lophocolea parva. VI: Campbell 1971 as Lophocolea parva, Miller et al. 1983 as Lophocolea parva.

** C. samoanus J.J.Engel et R.M.Schust. TA: Campbell 1971 as Lophocolea rectangulata. VI: Campbell 1971 as Lophocolea rectangulata, Miller et al. 1983 as Lophocolea rectangulata.

\section{Cololejeunea}

$\star * * \quad$ C. aequabilis (Sande Lac.) Schiffn. TA: Pócs et al. 2011. VI: Pócs et al. 2011.

*** C. amphibola B.M.Thiers TA: Pócs et al. 2011. VI: Pócs et al. 2011.

*** C. angustiflora (Steph.) Mizut. TA: Pócs et al. 2011.

*** C. cardiocarpa (Mont.) A.Evans VI: Pócs et al. 2011.

** C. ceatocarpa (Ångstr.) Steph. TA: Pócs et al. 2011. VI: Hürlimann 1987.

*** C. cocoscola Tixier KA: Pócs et al. 2011. TA: Pócs et al. 2011. VI: Pócs et al. 2011.

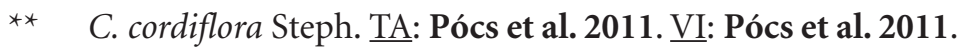

* ${ }^{3}$ C. decliviloba Steph. TA: Campbell 1971, Miller et al. 1983.

$* * * \quad$ C. diaphana A.Evans TA: Pócs et al. 2011.

*** C. equialbi Tixier KA: Pócs et al. 2011. TA: Pócs et al. 2011.

*** C. falcata (Horik.) Benedix TA: Pócs et al. 2011. VA: Pócs et al. 2011. VI: Pócs et al. 2011.

*** C. floccosa (Lehm. et Lindenb.) Schiffn. FIJ: Pócs \& Streimann 1999 [referring to Miller et al. 1983 who do not report it from Fiji]. TA: Pócs et al. 2011.

** C. haskarliana (Lehm.) Schiffn. var. haskarliana TA: Pócs et al. 2011. VI: Hürlimann 1987 as Cololejeunea hispidissima.

** C. hildebrandii (Austin) Steph. TA: Campbell 1971, Miller et al. 1983. VI: Campbell 1971, Miller et al. 1983.

3 Cololejeunea decliviloba is taxonomically doubtful and most likely should be placed in synonymy (Söderström et al. 2010). 
** C. hirta Steph. TA: Pócs et al. 2011.

*** C. huerlimannii Tixier TA: Pócs et al. 2011.

*** C. inflectens (Mitt.) Benedix KA: Pócs et al. 2011. TA: Pócs et al. 2011. VI: Pócs et al. 2011.

* $\quad{ }^{4}$ C. kapingaensis H.A.Mill. VI: Pócs et al. 2011.

*** C. kulenensis Tixier KA: Pócs et al. 2011. TA: Pócs et al. 2011. VI: Pócs et al. 2011.

** C. lanciloba Steph. KA: Pócs et al. 2011. TA: Pócs et al. 2011. VI: Pócs et al. 2011.

* C. latilobula (Herzog) Tixier KA: Pócs et al. 2011.

*** C. longifolia (Mitt.) Benedix ex Mizut. VI: Pócs et al. 2011.

*** C. metzgeriopsis (K.I.Goebel) Gradst., R.Wilson, Ilk.-Borg. et Heinrichs FII: Eggers 2006 as Metzgeriopsis pusilla, Eggers \& Pócs 2010. TA: Campbell 1971 as Metzgeriopsis pusilla, Miller et al. 1983 as Metzgeriopsis pusilla. VI: Schuster 1969 as Metzgeriopsis pusilla, Campbell 1971 as Metzgeriopsis pusilla, Miller et al. 1983 as Metzgeriopsis pusilla, Gradstein et al. 2006.

*** C. minutissima (Sm.) Schiffn. VI: Hürlimann 1987.

*** C. obliqua (Nees et Mont.) Schiffn. TA: Pócs et al. 2011. VI: Campbell 1971 as Cololejeunea nymannii, Miller et al. 1983 as Cololejeunea nymannii.

* ${ }^{5}$ C. paucimarginata Tixier TA: Pócs et al. 2011.

*** C. peraffinis (Schiffn.) Schiffn. FII: Hürlimann 1987 [incl. C. ocelloides], Pócs et al. 1994, Zhu \& So 2001. OV: Campbell 1971. TA: Campbell 1971. VI: Campbell 1971.

** - var. peraffinis FII: Pócs \& Streimann 1999. OV: Miller et al. 1983 [incl. C. ocellata and C. ocelloides]. TA: Miller et al. 1983 [incl. C. ocellata and C. ocelloides].VI: Miller et al. 1983 [incl. C. ocellata and C. ocelloides].

** C. polyantha (Steph.) H.A.Mill. FII: SYNTYPE of Lejeunea polyantha, Mitten 1873 as Lejeunea polyantha, Stephani 1916 as Physocolea polyantha, Thiers 1983 as Lejeunea polyantha, Miller et al. 1983 also as Physocolea polyantha and Lejeunea polyantha, Geissler \& Bischler 1985 as Leptocolea vitiensis, 1987 as Lejeunea polyantha, 1989 as Physocolea polyantha, Tixier 1985. TA: Campbell 1971 as Leptocolea vitiensis, Miller et al. 1983 as Leptocolea vitiensis and Cololejeunea vitiensis.VI: Campbell 1971 as Leptocolea vitiensis, Miller et al. 1983 as Cololejeunea vitiensis and Leptocolea vitiensis.

*** C. pseudoserrata Tixier TA: Pócs et al. 2011.

*** C. raduliloba Steph. TA: Pócs et al. 2011.VI: Pócs et al. 2011.

*** C. schmidtii Steph. TA: Pócs et al. 2011. VI: Pócs et al. 2011.

${ }^{4}$ Cololejeunea kapingaensis is treated as a new synonym of Cololejeunea planissima (Mitt.) Abeyw. (widespread in east and south-east Asia and Micronesia) by Inoue \& Iwatsuki (1969) but Pócs et al. (2011) recognise it.

${ }^{5}$ C. paucimarginata may be a form of C. raduliloba (Söderström et al. 2010), but Pócs et al. (2011) recognise it. 


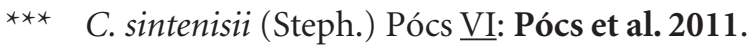

*** C. societatis Tixier TA: Pócs et al. 2011.

*** C. stylosa (Steph.) Steph. ex Mizut. VI: Pócs et al. 2011.

** C. trichomanis (Gottsche) Steph. FII: Pócs \& Ninh 2005. VA: Campbell 1971 as Leptocolea goebelii, Miller et al. 1983 as Cololejeunea goebelii and Leptocolea goebelii. VI: Campbell 1971 as Leptocolea goebelii, Miller et al. 1983 as Leptocolea goebelii.

** C. tridentata Tixier TA: Pócs et al. 2011.

** C. veillonii Tixier VI: Hürlimann 1987.

*** C. wightii Steph. KA: Pócs et al. 2011. TA: Pócs et al. 2011. VI: Pócs et al. 2011.

\section{Colura}

*** C. acroloba (Mont. ex Steph.) Ast FII: Gradstein et al. 2006, Wilson et al. 2007. VI: Pócs et al. 2011.

*** C. ari (Steph.) Steph. FII: Hürlimann 1987, Zhu \& So 2001, Eggers 2006, Eggers \& Pócs 2010. OV: Campbell 1971, Miller et al. 1983. TA: Campbell 1971, Miller et al. 1983, Pócs \& Eggers 2007. VI: Campbell 1971, Miller et al. 1983.

*** C. brevistyla Herzog TA: Pócs et al. 2011. VI: Pócs et al. 2011.

*** C. conica (Sande Lac.) K.I.Goebel FIJ: Hürlimann 1987, Pócs \& Streimann 1999, Zhu \& So 2001. OV: Campbell 1971 as Colura acutifolia, Miller et al. 1983. TA: Pócs \& Eggers 2007. VI: Pócs \& Eggers 2007.

*** C. corynophora (Nees, Lindenb. et Gottsche) Trevis. FII: Zhu \& So 2001. TA: Campbell 1971, Miller et al. 1983. VI: Jovet-Ast 1954, Campbell 1971, Miller et al. 1983.

$\star * * \quad$ C. crispiloba Ast VI: Pócs \& Eggers 2007.

*** C. cristata Ast KA: Pócs \& Eggers 2007.

** ${ }^{6}$ C. imperfecta Steph. FII: Gradstein et al. 2006, Wilson et al. 2007.

*** C. leratii (Steph.) Steph. FIJ: SYNTYPE of Lejeunea apiculata, Schiffner 1890 as Lejeunea apiculata, Bonner 1963b as Colurolejeunea apiculata, Miller et al. 1983 also as Colura apiculata and Lejeunea apiculata, Geissler \& Bischler 1987 as Lejeunea superba var. apiculata, Hürlimann 1987. TA: Pócs \& Eggers 2007. VI: Pócs \& Eggers 2007.

*** C. pluridentata Ast VI: Campbell 1971, Miller et al. 1983, Pócs \& Eggers 2007.

*** C. queenslandica B.M.Thiers VI: Pócs \& Eggers 2007.

*** C. superba (Mont.) Steph. FII: Mitten 1873 as Lejeunea superba, Miller et al. 1983. VI: Hürlimann 1987.

*** C. tenuicornis (A.Evans) Steph. VI: Pócs et al. 2011.

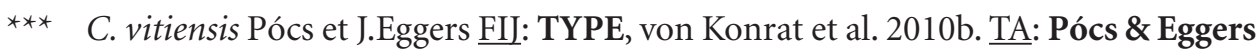
2007. VI: Pócs \& Eggers 2007.

${ }^{6}$ Colura imperfecta is very close to C. leratii (Söderström et al. 2010). 


\section{Conoscyphus}

*** C. trapezioides (Sande Lac.) Schiffn. VI: Hürlimann 1998.

\section{Cuspidatula}

*** C. contracta (Reinw., Blume et Nees) Steph. FIJ: Stephani 1897 as Anastrophyllum vitiense, Schiffner 1898, 1900 both as Anastrophyllum contractum, Bonner 1963b as Cuspidatula vitiensis, Grolle 1969 as Cuspidatula vitiensis, 1971, Bizot \& Pócs 1974, Váňa \& Piippo 1989b as Jamesoniella contracta, Váňa 1991b as Jamesoniella contracta, Schuster 2002. VI: TYPE of Anastrophyllum vitiense, Jack \& Stephani 1894 as Anastrophyllum vitiense, Stephani 1901 as Cuspidatula vitiensis, Bonner 1962b as Anastrophyllum vitiense, Hürlimann 1974, Miller et al. 1983 also as Anastrophyllum vitiense and Cuspidatula vitiensis.

\section{Dendrolejeunea}

*** D. fruticosa (Lindenb. et Gottsche) Lacout. FIJ: Verdoorn 1934 as Thysananthus fruticosus, Miller et al. 1983 as Dendrolejeunea vittata. VI: Jack \& Stephani 1894 as Thysanolejeunea fruticosa, Miller et al. 1983 as Thysananthus fruticosus.

\section{Denotarisia}

*** D. linguifolia (De Not.) Grolle FII: Schiffner 1898 as Jamesoniella ovifolia and Anastrophyllum graeffei, Stephani 1901 as Jamesoniella ovifolia, Grolle 1968 as Jamesoniella ovifolia, 1971, Váňa \& Piippo 1989b, Váňa 1991b, Schuster 2002. OV: TYPE of Anastrophyllum graeffei, Jack \& Stephani 1894 as Anastrophyllum graeffei, Bonner 1962b as Anastrophyllum graeffei, Miller et al. 1983 also as Jamesoniella ovifolia and Anastrophyllum graeffei. VI: Hürlimann 1989.

\section{Diplasiolejeunea}

*** D. cavifolia Steph. TA: Pócs et al. 2011. VI: Campbell 1971 as Diplasiolejeunea brachyclada, Miller et al. 1983.

\section{Drepanolejeunea}

*** D. angustifolia (Mitt.) Grolle VI: Hürlimann 1995.

*** D. dactylophora (Nees, Lindenb. et Gottsche) Schiffn. VI: Campbell 1971, Miller et al. 1983.

* $\quad{ }^{7}$ D. serricalyx Herzog FII: Pócs et al. 1994. VI: Campbell 1971, Miller et al. 1983.

* $\quad{ }^{8}$ D. tenera K.I.Goebel FII: Hürlimann 1995. VI: Campbell 1971, Miller et al. 1983.

*** D. ternatensis (Gottsche) Spruce ex Schiffn. FII: Mizutani 1978a, 1978b, Udar \& Awasthi 1982 as Drepanolejeunea ternatensis var. ternatensis, Miller et al. 1983 as Drepanolejeunea uncinata, Hürlimann 1995 as Drepanolejeunea ternatensis var. lancispina, Bapna \& Kachroo 2000b as Drepanolejeunea ternatensis var. ternatensis. OV: Jack \& Stephani 1894, Herzog 1939 as Drepanolejeunea ternatensis var. ternatensis, Miller et al. 1983 as Drepanolejeunea ternatensis var. ternatensis. VI:

${ }^{7}$ Drepanolejeunea serricalyx is doubtfully distinct from $D$. thwaitesiana (Mitt.) Steph. which is widespread in SE Asia (Söderström et al. 2010).

${ }^{8}$ Drepanolejeunea tenera is doubtfully distinct from $D$. pentadactyla (Mont.) Steph. which is widespread in the Paleotropics and reported from many areas in the Pacific, but not yet from Fiji (Söderström et al. 2010). 
Campbell 1971 as Drepanolejeunea ternatensis var. lancispina, Miller et al. 1983 as Drepanolejeunea ternatensis var. lancispina and var. ternatensis, Hürlimann 1995 as Drepanolejeunea ternatensis var. ternatensis.

** D. tricornua Herzog TA: Pócs et al. 2011.

*** D. vesiculosa (Mitt.) Steph. VI: Campbell 1971, Miller et al. 1983.

\section{Dumortiera}

*** ${ }^{9} D$. hirsuta (Sw.) Nees FIJ: Mitten 1873 as Dumortiera trichocephala, Miller et al. 1983 as Dumortiera trichocephala. OV: Schiffner 1890. TA: Campbell 1971, Miller et al. 1983 as Dumortiera hirsuta var. hirsuta. VI: Gibbs 1909 as Dumortiera trichocephala and Dumortiera velutina, Campbell 1971, Miller et al. 1983 as Dumortiera hirsuta var. hirsuta.

\section{Frullania}

** F. ampullifera J.B.Jack et Steph. FIJ: Koike 1994, Hentschel et al. 2009. KA: TYPE, Jack \& Stephani 1894, Stephani 1894, 1910, Bonner 1965, Pócs 2008b. OV: Verdoorn 1930, Hattori 1981, Miller et al. 1983, Pócs 2008b. TA: Pócs 2008b. VI: Campbell 1971, Miller et al. 1983, Hattori 1985, Pócs 2008b.

** F. angustistipa Steph. KA: Pócs $\mathbf{2 0 0 8 b}$ as Frullania angulistipa.

*** F. apiculata (Reinw., Blume et Nees) Nees FII: Hentschel et al. 2009. KA: Pócs 2008b. OV: Jack \& Stephani 1894, Campbell 1971. TA: Verdoorn 1937, Pócs 2008b. VI: Hattori 1985.

** - var. apiculata FII: Mitten 1873 as Frullania pacificae ["pacifica"], Stephani 1897, 1916 both as Frullania pacificae. OV: Miller et al. 1983. TA: Miller et al. 1983. VI: Miller et al. 1983 also as Frullania pacificae,.

** _ var. goebelii Schiffn. OV: Campbell 1971, Miller et al. 1983. TA: Campbell 1971, Miller et al. 1983. VI: Campbell 1971, Miller et al. 1983.

*** F. arecae (Spreng.) Gottsche var. arecae FIJ: Yuzawa 1991.

** F. auriculata S.Hatt. FII: Pócs 2008b. VI: TYPE, Hattori 1985, Engel 1992, Crosby \& Engel 2006.

** F. baladina Gottsche ex Steph. FIJ: Hentschel et al. 2009. KA: Pócs 2008b. VI: Pócs 2008b.

** F. capillaris Steph. FIJ: Pócs 2008b. VI: Hattori 1985.

$* * * \quad$ F. chevalieri (R.M.Schust.) R.M.Schust. TA: Pócs 2008b.

*** F. cordistipula (Reinw., Blume et Nees) Dumort. FII: Mitten 1862.

** F. deflexa Mitt. FIJ: TYPE, TYPE of Frullania pendula, Mitten 1862, Austin 1872 as Frullania pendula, Mitten 1873, Stephani 1911, 1916, Verdoorn 1930, Bonner 1965 also as Frullania pendula, Hattori 1977, 1980, 1986a, Thiers 1983, Miller et al. 1983 as Frullania pendula. OV: TYPE of Frullania varians, Jack \& Stephani 1894, Bonner 1965 as Frullania varians, Miller et al. 1983 also as Frullania varians. TA: Hattori 1980, Miller et al. 1983. VA: Campbell 1971, Miller et al. 1983. VI: Campbell 1971, Hattori 1980, 1985, Miller et al. 1983, Pócs 2008 b.

9 Dumortiera hirsuta is a variable species and recent studies (N.Salazar-Allen, pers. comm. 2009) indicate that more than one taxon may be involved. The most commonly recognised infrataxon is subsp. nepalensis (Taylor) R.M.Schust. and this is also reported from Fiji, but the taxonomy needs clarification. 


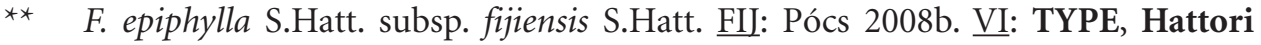
1985, Engel 1992, Crosby \& Engel 2006.

*** F. ericoides (Nees) Mont. FIJ: Stephani 1910 as Frullania vesiculosa, Bonner 1965 as Frullania vesiculosa, Miller et al. 1983 as Frullania vesiculosa. VI: Miller et al. 1983 as Frullania squarrosa [Viti Levu with a “?”], Pócs 2008b.

*** F. gaudichaudii (Nees et Mont.) Nees et Mont. FIJ: Hentschel et al. 2009. KA: Pócs 2008b. TA: Verdoorn 1937, Campbell 1971, Miller et al. 1983, Pócs 2008b. VA: Campbell 1971, Miller et al. 1983. VI: Hattori 1985, Hattori 1986a.

*** F. gracilis (Reinw., Blume et Nees) Gottsche, Lindenb. et Nees TA: Pócs 2008b.

** F. immersa Steph. FIJ: Pócs 2008b. VA: Verdoorn 1937, Campbell 1971, Miller et al. 1983. VI: Campbell 1971 as Frullania rechingeri, Miller et al. 1983 also as Frullania rechingeri, Hattori 1985, 1986a.

*** F. intermedia (Reinw., Blume et Nees) Dumort. FIJ: Clark \& Frye 1948, Verdoorn 1930, Bonner 1965, Inoue \& Miller 1965, Bapna \& Kachroo 2000b, Hentschel et al. 2009. KA: Verdoorn 1937, Miller et al. 1983. OV: Miller et al. 1983. TA: Miller et al. 1983. VI: Miller et al. 1983.

** - subsp.intermedia FII: TYPE of Frullania stellatitexta, Stephani 1911 as Frullania stellatitexta, Frullania angulosa and Frullania khedingiana ["kehdingiana"], Verdoorn 1930 as Frullania angulosa and Frullania stellatitexta, Bonner 1965 as Frullania khedingiana ["kedingiana"] and Frullania angulosa Mitt. KA: Campbell 1971 as Frullania intermedia f. intermedia. OV: Verdoorn 1928 as Frullania angulosa, Bonner 1965 as Frullania stellatitexta, Hattori 1980, Miller et al. 1983 as Frullania stellatitexta. TA: Hattori 1980. VI: Campbell 1971 as Frullania intermedia f. intermedia, Campbell 1971 as Frullania intermedia f. billardieriana, Hattori 1980, 1985, Miller et al. 1983 as Frullania intermedia f. billardieriana.

** - var. non-apiculata S.Hatt. FIJ: Hattori \& Streimann 1985, Hattori 1986a. TA: Pócs 2008b. VI: Hattori 1985.

*** F. meyeniana Lindenb. FIJ: Hentschel et al. 2009.

*** F. neurota Taylor FII: Pócs 2008b. VI: Campbell 1971, Miller et al. 1983.

*** F. nodulosa (Reinw., Blume et Nees) Nees FII: Mitten 1873, Schiffner 1898, Hentschel et al.2009. LA: Hattori 1980 as Frullania nodulosa var. nodulosa, Miller et al. 1983. VA: Hattori 1980 as Frullania nodulosa var. nodulosa. VI: TYPE of Frullania inflatistipula, Jack \& Stephani 1894, Verdoorn 1937, Bonner 1965 as Frullania inflatistipula, Campbell 1971, Hattori 1980 as Frullania nodulosa var. nodulosa, 1985, Miller et al. 1983 also as Frullania inflatistipula, Pócs 2008b.

** F. novocurvirostris S.Hatt. FII: Bonner 1965 as Frullania curvirostris, Hattori 1984, 1986a. KA: Pócs 2008b. OV: TYPE of Frullania curvatirostra, Jack \& Stephani 1894 as Frullania curvirostris, Stephani 1894 as Frullania curvirostris, 1910 as Frullania curvirostris and Frullania curvatirostra., Bonner 1965 as Frullania curvatirostra, Campbell 1971 as Frullania curvirostris, Hattori 1981, Miller et al. 1983 also as Frullania curvirostris, Pócs 2008b. VI: Campbell 1971 as Frullania curvirostris, Miller et al. 1983 also as Frullania curvirostris, Hattori 1985. 
** F. parhamii (R.M.Schust.) R.M.Schust. ex von Konrat, L.Söderstr. et A.Hagborg FI): Schuster 1964, 1969 both as Neohattoria parhamii, Hattori et al. 1977, 1986a both as Schusterella parhamii, Geissler \& Bischler 1990 as Schusterella parhamii, Pócs 2008b, Hentschel et al. 2009. VA: TYPE of Neohattoria parhamii, Schuster 1963, 1970 both as Neohattoria parhamii, Hattori et al. 1972 as Schusterella parhamii, Miller et al. 1983 as Schusterella parhamii and Neohattoria parhamii, Geissler \& Bischler 1989 as Neohattoria parhamii, von Konrat et al. 2006 as Neohattoria parhamii.

*** F. ramuligera (Nees) Mont. FII: Hattori \& Lin 1985 [with a “?”], Bai 2002. VI: Campbell 1971, Miller et al. 1983.

* ${ }^{10}$ F. serrata Gottsche $\underline{\text { FIJ: }}$ TYPE of Frullania grossispica, TYPE of Frullania meteoroides, Mitten 1862, 1873 both as Frullania meteoroides, Stephani 1897 as Frullania meteoroides, 1911 as Frullania meteoroides and Frullania grossispica, 1916 as Frullania meteoroides, Verdoorn 1930, Bonner 1965 as Frullania grossispica and Frullania meteoroides, Hattori 1973, Thiers 1983 as Frullania meteoroides, Miller et al. 1983 as Frullania meteoroides, Pócs 2008b, Singh et al. 2008. OV: Jack \& Stephani 1894 as Frullania meteoroides, Miller et al. 1983. VI: Verdoorn 1937, Campbell 1971, Miller et al. 1983 also as Frullania grossispica [Viti Levu with a “?”], Hattori 1985.

*** F. ternatensis Gottsche FII: Hattori 1982, Hattori \& Streimann 1985, Hattori \& Piippo 1986. TA: Pócs 2008b. VI: Hattori 1975b, Miller et al. 1983, Pócs 2008b.

** — var. non-appendiculata S.Hatt. FIJ: Hattori 1988. VI: Hattori 1985.

** — var. ternatensis FIJ: Hattori 1985.

** F. trichodes Mitt. FIJ: TYPE, Mitten 1862, 1873, Bonner 1965, Thiers 1983, Hattori \& Piippo 1986, Hattori 1988. KA: Pócs 2008b. VI: Miller et al. 1983 [Viti Levu with a "?"], Hattori 1985 as Frullania vethii, Pócs 2008 b.

** F. vaga Mitt. FIJ: TYPE, Mitten 1873, Stephani 1911, Verdoorn 1930, Bonner 1965, Hattori 1986b, Pócs 2008b. VI: Miller et al. 1983 [Viti Levu with a “?”].

** Fivipara Pócs FIJ: von Konrat et al. 2010b. KA: TYPE, Pócs 2008b.

\section{Harpalejeunea}

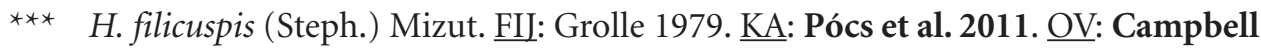
1971 as Drepanolejeunea riddleana, Miller et al. 1983 as Drepanolejeunea riddleana and Drepanolejeunea filicuspis. TA: Pócs et al. 2011. VI: Pócs et al. 2011.

* ${ }^{11}$ H. vitrea Herzog VA: TYPE, Herzog 1951a, Miller et al. 1983, Grolle \& ReinerDrehwald 1999.

${ }^{10}$ Frullania serrata is probably conspecific with F. cordistipula. The two differ only by the degree of dentation of the female bracts (Söderström et al. 2010).

${ }^{11}$ Harpalejeunea vitrea is a Lejeunea sp. (non L. vitrea Nees et al.) according to Grolle \& Reiner-Drehwald (1999). 


\section{Herbertus}

** H. longifissus Steph. VI: Miller 1968 also as Herbertus tutuilanus, Miller et al. 1983 also as Herbertus tutuilanus.

\section{Heteroscyphus}

*** H. argutus (Reinw., Blume et Nees) Schiffn. FII: Mitten 1861 as Chiloscyphus argutus, Stephani 1897 as Chiloscyphus argutus, Piippo 1985. OV: Jack \& Stephani 1894 as Chiloscyphus argutus, Miller et al. 1983. VA: Campbell 1971, Miller et al. 1983. VI: Gibbs 1909 as Chiloscyphus argutus, Campbell 1971, Miller et al. 1983.

* - var. argutus FIJ: Mitten 1873 as Chiloscyphus endlicherianus.

*** H. aselliformis (Reinw., Blume et Nees) Schiffn. FII: SYNTYPE of Chiloscyphus confluens, Mitten 1873 as Chiloscyphus confluens, Stephani 1897, 1916 both as Chiloscyphus confluens, Bonner 1963b as Chiloscyphus confluens, Grolle 1965 as Chiloscyphus aselliformis, Kitagawa 1979, 1981 both as Chiloscyphus aselliformis, Grolle 1980, Thiers 1983 as Chiloscyphus confluens, Piippo 1985, 1989b, Hürlimann 1998, Yamada \& Hayashi 2003. OV: Jack \& Stephani 1894 as Chiloscyphus confluens, Campbell 1971 as Heteroscyphus confluens, Miller et al. 1983 as Heteroscyphus confluens. VI: Campbell 1971, Miller et al. 1983 also as Chiloscyphus aselliformis, Hürlimann 1998.

$\star * * \quad$ H. coalitus (Hook.) Schiffn. FII: Piippo 1985. VI: Campbell 1971 as Heteroscyphus communis, Miller et al. 1983 as Heteroscyphus communis and Chiloscyphus communis.

** H. graeffei (J.B.Jack et Steph.) Grolle FII: Stephani 1897 as Lophocolea graeffei, Engel \& Schuster 1985 ad Chiloscyphus graeffei. OV: Campbell 1971 as Lophocolea graeffei, Miller et al. 1983 as Lophocolea graeffei. VI: SYNTYPE of Lophocolea graeffei, Jack \& Stephani 1894 as Lophocolea graeffei, Campbell 1971 as Lophocolea graeffei, Miller et al. 1983 as Lophocolea graeffei, Geissler \& Bischler 1985 as Lophocolea graeffei.

*** H. succulentus (Gottsche) Schiffn. FIJ: Miller et al. 1983 as Heteroscyphus concinnus. TA: Campbell 1971 as Heteroscyphus concinnus, Miller et al. 1983 also as Chiloscyphus succulentus.

\section{Hymenophyton}

*** H. flabellatum (Labill.) Dumort. ex Trevis. FIJ: Evans 1925, Arnell 1958, Schuster 1969, Miller et al. 1983, Furuki 2002, Pfeiffer 2000.

\section{Jamesoniella}

$\star \star \star 12 \mathrm{~J}$. flexicaulis (Nees) Schiffn. FIJ: Mitten 1873 as Jungermannia flexicaulis, Miller et al. 1983 as Crossogyna flexicaulis. VA: Campbell 1971. VI: Campbell 1971, Hürlimann 1974.

\section{Jubula}

** J. hutchinsiae (Steph.) Verd. subsp. javanica (Steph.) Verd. VI: Campbell 1971, Miller et al. 1983.

${ }^{12}$ The genus Jamesoniella was transferred to Syzygiella and Cuspidatula by Feldberg et al. (2010) but J. flexicaulis was not included in their study and they did not transfer it. 


\section{Kurzia}

*** K. gonyotricha (Sande Lac.) Grolle VA: Pócs et al. 2011.

\section{Lejeunea}

$\star * \star \quad$ L. alata Gottsche TA: Pócs et al. 2011. VI: Pócs et al. 2011.

*** L. anisophylla Mont. KA: Pócs et al. 2011. TA: Pócs et al. 2011. VI: Pócs et al. 2011.

** L. apiculata Sande Lac. VI: Pócs et al. 2011.

*** L. capensis Gottsche FIJ: Schuster 1984 as Lejeunea caespitosa, Hürlimann 1993 as Lejeunea caespitosa. VI: Jones 1972 as Lejeunea caespitosa, Schuster 1980b as Lejeunea caespitosa, Miller et al. 1983 as Lejeunea caespitosa.

** L. caviloba (Steph.) Steph. ex Besch. VI: Hürlimann 1993.

** L. cocoes Mitt. KA: Pócs et al. 2011. TA: Pócs et al. 2011. VI: Pócs et al. 2011.

** L. crassiretis Mitt. FIJ: TYPE, Mitten 1873, Stephani 1915, Thiers 1983, Geissler \& Bischler 1987. VI: Campbell 1971, Miller et al. 1983.

** L. cuculliflora (Steph.) Mizut. FII: Stephani 1896, 1914 both as Taxilejeunea cuculliflora, Mizutani 1978a, Geissler \& Bischler 1987, 1990 as Taxilejeunea cuculliflora, Hürlimann 1995. OV: TYPE of Taxilejeunea cuculliflora, Eifrig 1937 as Taxilejeunea cuculliflora, Mizutani 1976, Grolle 1979, Miller et al. 1983 also as Taxilejeunea cuculliflora, Grolle 1988. VA: Campbell 1971 as Taxilejeunea cuculliflora, Miller et al. 1983 also as Taxilejeunea cuculliflora. VI: Campbell 1971 as Taxilejeunea cuculliflora, Miller et al. 1983 also as Taxilejeunea cuculliflora.

*** L. discreta Lindenb. TA: Pócs et al. 2011. VI: Hürlimann 1993.

*** L. exilis (Reinw., Blume et Nees) Grolle TA: Pócs et al. 2011. VI: Pócs et al. 2011.

*** L. flava (Sw.) Nees FIJ: Mitten 1873 as Lejeunea uvifera, Stephani 1897 as Euosmolejeunea uvifera, Miller et al. 1983 as Euosmolejeunea uvifera. OV: Campbell 1971, Miller et al. 1983. TA: Pócs et al. 2011. VI: Campbell 1971, Miller et al. 1983.

** - subsp. orientalis R.M.Schust. VI: Hürlimann 1993.

** L. gracilipes (Taylor) Spruce TA: Campbell 1971 as Taxilejeunea gracilipes, Miller et al. 1983 also as Taxilejeunea gracilipes. VA: Campbell 1971 as Taxilejeunea gracilipes, Miller et al. 1983 also as Taxilejeunea gracilipes. VI: Campbell 1971 as Taxilejeunea gracilipes, Miller et al. 1983 also as Taxilejeunea gracilipes.

*** L. lumbricoides (Nees) Nees OV: TYPE of Hygrolejeunea graeffeana ["Samoa, Ovalau"], Stephani 1914 as Hygrolejeunea graeffeana ["Samoa, Ovalau"], Eifrig 1937 as Taxilejeunea lumbricoides, Bonner 1966 as Hygrolejeunea graeffeana ["Samoa, Ovalau"], Grolle \& Schultze-Motel 1973, Miller et al. 1983 also as Hygrolejeunea graeffeana. VI: Hürlimann 1993.

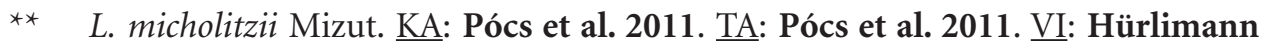
1993.

** L. microloba Taylor OV: Grolle 1979, Miller et al. 1983.

** L. mimula Hürl. FII: Hürlimann 1995. VI: Hürlimann 1993.

** L. nietneri (Steph.) Steph. VI: Campbell 1971, Miller et al. 1983.

** L. patersonii (Steph.) Steph. OV: TYPE of Microlejeunea samoana, Miller et al. 1963b, 1983 both as Microlejeunea samoana Steph. VI: Hürlimann 1993. 
** L. procumbens Mitt. FII: TYPE, Mitten 1873, Stephani 1914 as Hygrolejeunea procumbens, Bonner 1965 as Hygrolejeunea procumbens, Thiers 1983, Geissler \& Bischler 1987. VI: Miller et al. 1983 also as Hygrolejeunea procumbens [Viti Levu with a "?"].

* ${ }^{13}$ L. sinclairii Mitt. FIJ: TYPE, Mitten 1862, Mitten 1873 as Bryopteris sinclairii, Stephani 1911 as Thysananthus sinclairii, Bonner 1963a as Bryopteris sinclairii, Thiers 1983, Miller et al. 1983 also as Lejeunea sinclairii and Thysananthus sinclairii, Geissler \& Bischler 1987, 1990 as Thysananthus sinclairii.

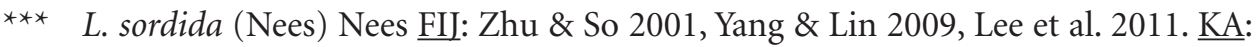
Pócs et al. 2011. TA: Pócs et al. 2011. VI: Hürlimann 1993.

** L. subigiensis (Steph.) Steph. OV: Campbell 1971, Miller et al. 1983. VI: Campbell 1971, Miller et al. 1983.

*** L. umbilicata (Nees) Nees FIJ: Hürlimann 1995. OV: Jack \& Stephani 1894 as Taxilejeunea umbilicata, Eifrig 1937 as Taxilejeunea umbilicata, Miller et al. 1983. TA: Pócs et al. 2011. VI: Jack \& Stephani 1894 as Taxilejeunea umbilicata, Miller et al. 1983.

\section{Lepicolea}

*** L. rara (Steph.) Grolle FIJ: Grolle 1965, 1968 both as Lepicolea loriana, Miller et al. 1983 as Lepicolea loriana, Piippo 1984a, Schuster 2000. TA: Pócs et al. 2011. VI: Hürlimann 1983 as Lepicolea loriana.

\section{Lepidolejeunea}

*** L. bidentula (Steph.) R.M.Schust. FII: SYNTYPE of Hygrolejeunea decurrens, Stephani 1896 as Hygrolejeunea decurvifolia, 1914 as Pycnolejeunea bidentula, Jovet-Ast \& Tixier 1962 as Pycnolejeunea bidentula, Bonner 1966 as Hygrolejeunea decurvifolia, Mizutani 1967 as Pycnolejeunea bidentula [with a “?”], Tixier 1970 as Pycnolejeunea bidentula, Miller et al. 1983 as Hygrolejeunea decurvifolia, Geissler \& Bischler 1989 as Pycnolejeunea decurvifolia, Zhu \& So 2001. KA: Pócs et al. 2011. OV: Jack \& Stephani 1894 as Pycnolejeunea bidentula, Miller et al. 1983 as Pycnolejeunea bidentula. TA: Pócs et al. 2011. VI: Campbell 1971 as Pycnolejeunea bidentula, Miller et al. 1983 as Pycnolejeunea bidentula, Hürlimann 1995.

*** — var. bidentula OV: Piippo 1986.

*** L. borneensis (Steph.) R.M.Schust. TA: Pócs et al. 2011. VI: Pócs et al. 2011.

*** L. graeffei (J.B.Jack et Steph.) R.M.Schust. FII: Stephani 1911 as Archilejeunea graeffei, Horikawa 1943 as Pycnolejeunea graeffei, Geissler \& Bischler 1987 as Lejeunea graeffei, 1989 as Pycnolejeunea graeffei. OV: TYPE of Archilejeunea graeffei, Jack \& Stephani 1894 as Archilejeunea graeffei, Bonner 1962b as Archilejeunea graeffei, Mizutani 1972 as Pycnolejeunea graeffei, Miller et al. 1983 as Pycnolejeunea graeffei and Archilejeunea graeffei, Piippo 1986, TA: Pócs et al. 2011. VI: Hürlimann 1995.

*** L.integristipula(J.B.Jack et Steph.) R.M.Schust. FIJ:Stephani 1896 as Hygrolejeunea

${ }^{13}$ Lejeunea sinclairii may be conspecific with L. flava (Grolle 1982). It is treated as a synonym of Caudalejeunea reniloba and Thysananthus fruticosus (=Dendrolejeunea fruticosa) by Miller et al. (1983). 
devexiloba, Inoue \& Miller 1965 as Hygrolejeunea devexiloba, Mizutani 1978a both as Pycnolejeunea integristipula, Miller et al. 1983 as Hygrolejeunea devexiloba, Geissler \& Bischler 1990 as Symbiezidium integristipulum, Wilson et al. 2007. KA: Pócs et al. 2011. OV: TYPE of Pycnolejeunea integristipula, Jack \& Stephani 1894 as Pycnolejeunea integristipula, Stephani 1912 as Symbiezidium integristipulum, Campbell 1971 as Symbiezidium integristipulum, Mizutani 1972 as Pycnolejeunea integristipula, Miller et al. 1983 as Pycnolejeunea integristipula and Symbiezidium integristipulum, Piippo 1986, Geissler \& Bischler 1987 as Lejeunea integristipula, 1989 as Pycnolejeunea integristipula, Pócs et al. 2011. TA: Pócs et al. 2011. VI: Campbell 1971 as Symbiezidium integristipulum, Miller et al. 1983 as Symbiezidium integristipulum and Pycnolejeunea integristipula.

*** L. involuta (Gottsche) Grolle FII: Mitten 1873 as Lejeunea hawaiiensis, Miller et al. 1983 as Harpalejeunea owaihiensis.

\section{Leptolejeunea}

*** L. elliptica (Lehm. et Lindenb.) Schiffn. FII: Schuster 1984, Hürlimann 1995 as Leptolejeunea subacuta, Pócs \& Streimann 1999 as Leptolejeunea subacuta. TA: Campbell 1971 as Leptolejeunea subacuta, Miller et al. 1983 as Leptolejeunea subacuta. VI: Campbell 1971 as Leptolejeunea subacuta, Miller et al. 1983 as Leptolejeunea subacuta.

*** L. epiphylla (Mitt.) Steph. VA: Miller et al. 1983 as Lejeunea polyrhiza. VI: Campbell 1971, Miller et al. 1983.

** L. maculata (Mitt.) Schiffn. FII: Pócs \& Streimann 1999 as Leptolejeunea rhombifolia, Eggers \& Pócs 2010 as Leptolejeunea rhombifolia. OV: Campbell 1971 as Leptolejeunea schiffneri and Leptolejeunea radiata, Miller et al. 1983 as Leptolejeunea radiata and Leptolejeunea schiffneri. TA: Campbell 1971 as Leptolejeunea radiata and Leptolejeunea schiffneri, Miller et al. 1983 as Leptolejeunea radiata and Leptolejeunea schiffneri. VA: Campbell 1971 as Leptolejeunea radiata, Miller et al. 1983 as Leptolejeunea radiata. VI: Campbell 1971 as Leptolejeunea schiffneri, Leptolejeunea radiata and Leptolejeunea rhombifolia, Miller et al. 1983 as Leptolejeunea rhombifolia, Leptolejeunea radiata and Leptolejeunea schiffneri, Hürlimann 1995.

** L. tripuncta (Mitt.) Steph. FII: TYPE of Lejeunea tripuncta, Mitten 1873 as Lejeunea tripuncta, Stephani 1913, Thiers 1983 as Lejeunea tripunctata, Miller et al. 1983 as Lejeunea tripuncta, Geissler \& Bischler 1985, 1987 as Lejeunea tripuncta Mitt. VI: Miller et al. 1983 [Viti Levu with a “?”].

\section{Lobatiriccardia}

*** L. coronopus (De Not. ex Steph.) Furuki OV: Campbell 1971 as Riccardia lobata, Miller et al. 1983 as Riccardia lobata var. lobata. VI: Gibbs 1909 as Aneura lobata, Campbell 1971 as Riccardia lobata, Miller et al. 1983 as Riccardia lobata var. lobata.

\section{Lopholejeunea}

*** L. eulopha (Taylor) Schiffn. FIJ: Mitten 1862 as Lejeunea eulopha, 1873 as Phragmicoma eulopha, Stephani 1912 as Lopholejeunea immersa, Verdoorn 1934, Miller et al. 1983 as Lopholejeunea immersa, Sass-Gyarmati 2008. KA: Pócs et al. 
2011. TA: Pócs et al. 2011. VI: Campbell 1971, Miller et al. 1983, Hürlimann 1991.

*** L. hispidissima Steph. TA: Pócs et al. 2011. VI: Pócs et al. 2011.

*** L. nigricans (Lindenb.) Schiffn. FIJ: Stephani 1912 as Lopholejeunea multiflora, Sass-Gyarmati 2008. KA: Pócs et al. 2011. OV: TYPE of Lopholejeunea multiflora, Jack \& Stephani 1894 as Lopholejeunea multiflora, Miller et al. 1983 as Lopholejeunea multiflora, Geissler \& Bischler 1985 as Lopholejeunea multiflora, Zhu \& Gradstein 2005. TA: Pócs et al. 2011. VI: Campbell 1971 as Lopholejeunea multiflora, Miller et al. 1983 as Lopholejeunea multiflora.

** L. pocsii Gyarmati FIJ: Sass-Gyarmati 2008. VI: TYPE, Sass-Gyarmati 2005.

** L. renistipula (Mitt.) Steph. FII: TYPE of Phragmicoma renistipula, Mitten 1873 as Phragmicoma renistipula, Stephani 1912, Thiers 1983 as Phragmicoima renistipula, Miller et al. 1983 also as Phragmicoma renistipula, Geissler \& Bischler 1985, 1989 as Phragmicoma renistipula.

*** L. subfusca (Nees) Schiffn. FIJ: Sass-Gyarmati 2008. KA: Pócs et al. 2011. TA: Pócs et al. 2011. VA: Campbell 1971, Miller et al. 1983. VI: Campbell 1971, Miller et al. 1983, Hürlimann 1991.

** L. vojtkoana Gyarmati FII: von Konrat et al. 2010b. KA: TYPE, Sass-Gyarmati 2008. TA: Sass-Gyarmati 2008.

*** L. zollingeri (Steph.) Schiffn. FIJ: Sass-Gyarmati 2005, 2008, Haerida et al. 2010. VA: Zhu \& Gradstein 2005.

\section{Marchantia}

*** M. vitiensis Steph. FII: TYPE, SYNTYPE of Marchantia samoana Steph., Stephani 1899a, also as Marchantia samoana, Grolle 1968 as Marchantia samoana, Geissler \& Bischler 1985 as Marchantia samoana. OV: Geissler \& Bischler 1985, BischlerCausse 1989. VA: Campbell 1971, Miller et al. 1983, Bischler-Causse 1989. VI: Gibbs 1909, Hürlimann 1960, 1976 both as Marchantia samoana, Campbell 1971, Miller et al. 1983 also as Marchantia samoana, Bischler-Causse 1989.

\section{Mastigolejeunea}

*** M. auriculata (Wilson) Schiffn. FII: Verdoorn 1934 as Mastigolejeunea humilis, Miller et al. 1983 as Mastigolejeunea guahamensis. OV: Jack \& Stephani 1894 as Mastigolejeunea guahamensis, Miller et al. 1983 as Mastigolejeunea humilis.

*** M. calcarata (Mitt. ex Steph.) Verd. FIJ: Stephani 1911 as Archilejeunea calcarata, Verdoorn 1934, Bonner 1962b as Archilejeunea calcarata, Thiers 1983 as Phragmicoma calcarata, Geissler \& Bischler 1985, Hürlimann 1989, Sass-Gyarmati 2003. KA: Pócs et al. 2011. OV: TYPE of Phragmicoma calcarata, Mitten 1873 as Phragmicoma calcarata, Miller et al. 1983 also as Archilejeunea calcarata and Phragmicoma calcarata, Thiers 1985, Geissler \& Bischler 1989 as Phragmicoma calcarata, Thiers \& Gradstein 1989,Pócs et al. 2011. VI: Campbell 1971, Miller et al. 1983 .

\section{Mastigophora}

*** M. diclados (Brid. ex F.Weber) Nees FIJ: Miller et al. 1983 as Mastigophora angustisecta. OV: Jack \& Stephani 1894, Campbell 1971, Miller et al. 1983. TA: Campbell 1971, Miller et al. 1983. VA: TYPE of Mastigophora angustisecta, 
Herzog 1951 b as Mastigophora angustisecta, Miller et al. 1983, Geissler \& Bischler 1985 as Mastigophora angustisecta. VI: Campbell 1971, Hürlimann 1983, Miller et al. 1983.

\section{Metalejeunea}

** M. crassitexta (J.B.Jack et Steph.) Pócs FIJ: Schuster 1963 as Lejeunea crassitexta, Miller et al. 1983 as Lejeunea crassitexta, Geissler \& Bischler 1987 as Lejeunea crassitexta. KA: Pócs et al. 2011. OV: TYPE of Microlejeunea crassitexta, Jack \& Stephani 1894 as Microlejeunea crassitexta, Stephani 1915 as Microlejeunea crassitexta, Miller et al. 1963b, 1983 both as Microlejeunea crassitexta, Campbell 1971 as Microlejeunea crassitexta, Geissler \& Bischler 1985 as Microlejeunea crassitexta, Pócs et al. 2011. TA: Pócs et al. 2011. VI: Campbell 1971 as Microlejeunea crassitexta, Miller et al. 1983 as Microlejeunea crassitexta, Pócs et al. 2011.

*** M.cucullata (Reinw., Blume et Nees) Grolle FII: Bischler et al. 1962 as Microlejeunea cucullata., Miller et al. 1963a as Microlejeunea cucullata, Inoue \& Miller 1965 as Microlejeunea cucullata, Pócs et al. 1967 as Microlejeunea cucullata, Mizutani 1978a as Lejeunea cucullata. KA: Pócs et al. 2011. OV: Jack \& Stephani 1894 as Microlejeunea albicans, Miller et al. 1963b, 1983 both as Microlejeunea cucullata. VI: Campbell 1971 as Microlejeunea cucullata, Miller et al. 1983 as Microlejeunea cucullata, Hürlimann 1993 as Lejeunea cucullata.

\section{Metzgeria}

***14 M. ciliata Raddi FIJ: Piippo 1991 as Metzgeria decipiens, Kuwahara 1984, 1985 both as Metzgeria decipiens, Koponen et al. 2000 as Metzgeria decipiens, Costa 2008. OV: Campbell 1971 as Metzgeria decipiens, Miller et al. 1983 as Metzgeria decipiens.VI: Hürlimann 1969 as Metzgeria decipiens, Campbell 1971 as Metzgeria decipiens, Miller et al. 1983 as Metzgeria decipiens.

*** M. furcata (L.) Corda FII: Mitten 1873, Kuwahara 1966, 1985 both as Metzgeria densiseta. OV: Jack \& Stephani 1894, Miller et al. 1983 [with a “?”]. TA: Pócs et al. 2011. VI: Gibbs 1909, Campbell 1971 as Metzgeria densiseta, Miller et al. 1983 also as Metzgeria densiseta Steph.

*** M. leptoneura Spruce FII: SYNTYPE of Metzgeria papulosa, Stephani 1899d as Metzgeria papulosa, Kuwahara 1961, 1966, 1968, 1969 all as Metzgeria parvipapulosa, 1982, 1984 both as Metzgeria papulosa, 1985 also as M. papulosa, Engel 1976, Miller et al. 1983 as Metzgeria parvipapulosa, Schuster 1992a as Metzgeria parvipapulosa, Damsholt 2002. VI: Kuwahara 1966 as Metzgeria hamata, Campbell 1971 as Metzgeria hamata, Miller et al. 1983, Piippo 1991.

** — var. leptoneura FIJ: Schuster 1992a. VI: Campbell 1971 as Metzgeria hamata.

** M. lindbergii Schiffn. FII: Kuwahara 1961 as Metzgeria oceanica, 1965, 1966, 1968 all as Metzgeria pectinata, 1985 as Metzgeria minor, Miller et al. 1983 as Metzgeria pectinata and Metzgeria oceanica.

${ }^{14}$ Metzgeria ciliata was (as $M$. decipiens) treated as a synonym of $M$. furcata by Grolle (2002b) but accepted by Costa (2008). Some reports of M. furcata may belong here. 


\section{Microlejeunea}

** M. lunulatiloba Horik. VI: Pócs et al. 2011.

\section{Mnioloma}

** M. novaezelandiae J.J.Engel VI: Pócs et al. 2011.

\section{Notoscyphus}

*** N. lutescens (Lehm. et Lindenb.) Mitt. VI: Schuster 2002.

\section{Pallavicinia}

*** P. lyellii (Hook.) Carruth. FIJ: Stephani 1900, Bonner 1965 as Diplomitrion lyellii, Miller et al. 1983, Schuster 1992a.

\section{Phaeolejeunea}

*** P. amicorum (Hürl.) Pócs FIJ: Wilson et al. 2007. KA: Pócs 2008a, Pócs et al. 2011. TA: Pócs 2008a, Pócs et al. 2011. VI: Pócs et al. 2011.

** P. inermis (Steph.) Mizut. KA: Pócs et al. 2011.

\section{Plagiochila}

*** Pabietina (Nees) Nees et Mont. FII: Bonner 1962a, Miller et al. 1983, Inoue 1986, Piippo 1989a. VI: Hürlimann 1974, Inoue 1984.

*** P. arbuscula (Brid. ex Lehm. et Lindenb.) Lindenb. FII: Mitten 1861, Mitten 1873, Stephani 1903b as Plagiochila belangeriana, Herzog 1938 as Plagiochila heterospina, Bonner 1962a as Plagiochila heterospina, Miller et al. 1983 as Plagiochila heterospina, Geissler \& Bischler 1989 as Plagiochila heterospina, Nair et al. 2005. OV: Jack \& Stephani 1894 as Plagiochila belangeriana, Campbell 1971 as Plagiochila belangeriana var. heterospina, Miller et al. 1983, So 2000. TA: HOLOTYPE of Plagiochila heterospina, Campbell 1971 as Plagiochila belangeriana var. heterospina, Miller et al. 1983, Inoue 1984. VA: Campbell 1971 as Plagiochila belangeriana var. heterospina, Miller et al. 1983, Inoue 1984. VI: Campbell 1971 as Plagiochila belangeriana var. heterospina, Hürlimann 1974 as Plagiochila alta, Miller et al. 1983, So 2000, 2001a, b.

*** P. bantamensis (Reinw., Blume et Nees) Mont. FII: Piippo 1989a as Plagiochila parvisacculata. VI: Campbell 1971 as Plagiochila auriculata, Miller et al. 1983 as Plagiochila auriculata, So \& Grolle 2000, So 2001 a.

** P. bicornuta Steph. TA: Pócs et al. 2011.

** P. chauviniana Mont. FII: Stephani 1904a as Plagiochila longispica, Miller et al. 1983 as Plagiochila longispica.

** P. fissidentoides Taylor FIJ: Stephani 1903a ["fissidentoidea"]. OV: Campbell 1971 ["fissidentoidea"], Miller et al. 1983. TA: Campbell 1971 ["fissidentoidea"], Miller et al. 1983. VI: Campbell 1971 [“fissidentoidea”], Miller et al. 1983.

*** P. hampeana Gottsche FII: Piippo 1989a, So \& Grolle 1999, So \& Grolle 2001. VI: Inoue 1970, Hürlimann 1974, Miller et al. 1983, Inoue 1984.

** P. huerlimannii Inoue FIJ: Inoue 1972c. VI: TYPE, Inoue 1970, Hürlimann 1974, Miller et al. 1983, Geissler \& Bischler 1989, Grolle \& So 1999, So 2001 b. 
*** P. javanica (Sw.) Nees et Mont. VI: Gibbs 1909 as Plagiochila powellii, Miller et al. 1983 as Plagiochila powellii.

*** P. sandei Dozy ex Sande Lac. FIJ: TYPE of Plagiochila seemannii and its var. perspinosa, Mitten 1861, 1873 both as Plagiochila seemannii, Stephani 1904a as Plagiochila seemannii, Herzog 1938 as Plagiochila seemannii var. perspinosa, Bonner 1962a as Plagiochila seemannii and its var. perspinosa, Thiers 1983 as Plagiochila seemannii, Miller et al. 1983 as Plagiochila seemannii var. perspinosa, Geissler \& Bischler 1989 as Plagiochila seemannii and its var. perspinosa, Piippo 1989a as Plagiochila seemannii, So \& Grolle 1999, So 2001b. OV: Campbell 1971 as Plagiochila seemannii, Miller et al. 1983 as Plagiochila seemannii. TA: Campbell 1971 as Plagiochila seemannii, Miller et al. 1983 as Plagiochila seemannii. VA: Grolle 1968 as Plagiochila seemannii, Miller et al. 1983 as Plagiochila seemannii. VI: Campbell 1971 as Plagiochila seemannii, Hürlimann 1974 as Plagiochila seemannii, Miller et al. 1983 as Plagiochila seemannii.

*** P. teysmannii Sande Lac. OV: TYPE of Plagiochila ovalava, Stephani 1903c as Plagiochila ovalava, Bonner 1962a as Plagiochila ovalava, Miller et al. 1983 as Plagiochila ovalava, Geissler \& Bischler 1989 as Plagiochila ovalava, So 2001 b.

** P. vitiensis Mitt. FIJ: Mitten 1873, Stephani 1903a as Plagiochila tahitensis, Bonner 1962a, Inoue 1981, Miller et al. 1983 as Plagiochila brotheri, Inoue 1986, Geissler \& Bischler 1989. VI: TYPE and TYPE of Plagiochila blepharophora \& vitilevuana, Mitten 1861, Schiffner 1890 as Plagiochila blepharophora $\varepsilon$ vitilevuana, Bonner 1962a as Plagiochila blepharophora $\varepsilon$ vitilevuana, Inoue 1970, Hürlimann 1974, Thiers 1983, Miller et al. 1983 also as Plagiochila tahitensis [with a "?"] and Plagiochila blepharophora var. vitilevuana, Scott \& Bradshaw 1985, Geissler \& Bischler 1989 as Plagiochila blepharophora \& vitilevuana, So 2001 b.

\section{Plagiochilion}

*** P. braunianum (Nees) S.Hatt. VI: Hürlimann 1974 [“braunianus”], Miller et al. 1983.

*** P. oppositum (Reinw., Blume et Nees) S.Hatt. FII: TYPE of Plagiochila geminifolia, Mitten 1873 as Plagiochila geminifolia, Stephani 1897 as Plagiochila geminifolia, 1904b as Plagiochila opposita, Schiffner 1898, 1900 both as Plagiochila opposita, Inoue 1958 as Noguchia opposita, 1982, Bonner 1962a as Plagiochila geminifolia, Thiers 1983 as Plagiochila geminifolia, Miller et al. 1983 as Plagiochila geminifolia, Geissler \& Bischler 1989 as Plagiochila geminifolia, So 2001b. OV: Jack \& Stephani 1894 as Plagiochila geminifolia, Miller et al. 1983. VI: Pócs et al. 2011.

\section{Pleurozia}

*** P. conchifolia (Hook. et Arn.) Austin

** — var. conchifolia TA: Pócs et al. 2011.

*** P. gigantea (F.Weber) Lindb. $\underline{\mathrm{OV}}$ : Jack \& Stephani 1894 as Physiotium giganteum, Miller et al. 1983 also as Physiotium giganteum, Thiers 1993. TA: Thiers 1993. VI: Gibbs 1909, Campbell 1971, Miller et al. 1983, Thiers 1993. 


\section{Plicanthus}

$\star * \star 15$ P. birmensis (Steph.) R.M.Schust. VI: Campbell 1971 as Chandonanthus birmensis, Miller et al. 1983 as Chandonanthus birmensis.

*** P. hirtellus (F.Weber) R.M.Schust. FIJ: Schuster 1963 as Chandonanthus hirtellus, Váňa \& Piippo 1989b as Chandonanthus hirtellus. VI: Miller et al. 1983 as Chandonanthus hirtellus [Viti Levu with a "?"].

\section{Porella}

${ }^{\star * \star}{ }^{16}$ P. elegantula (Mont.) E.A.Hodgs. FII: Mitten 1873 as Madotheca stangeri, Miller et al. 1983 as Madotheca stangeri and Porella stangeri. OV: Campbell 1971, Miller et al. 1983. VI: Campbell 1971, Miller et al. 1983.

** P. viridissima (Mitt.) Grolle FIJ: Stephani 1910 as Madotheca viridissima, Hattori 1975a as Porella viridissima var. viridissima. OV: Miller et al. 1983 as Porella viridissima var. viridissima, So 2002. VI: Campbell 1971 as Porella hebridensis, Hattori 1975d, Miller et al. 1983 as Porella viridissima var. viridissima and Porella hebridensis, Hattori 1985, 1986c, Hürlimann 1989, So 2002.

\section{Psiloclada}

*** P. clandestina Mitt. FII: Piippo 1984b, Pócs 1984. VA: Schuster 1970, Schuster 1963. VI: Schuster 1970, Campbell 1971.

** - subsp. melanesica R.M.Schust. II]: Schuster 2000. VI: Schuster 1980a, Miller et al. 1983.

\section{Ptychanthus}

*** P. striatus (Lehm. et Lindenb.) Nees FII: Mitten 1873 as Bryopteris striata. LA: Verdoorn 1937. TA: Pócs et al. 2011. VI : Gibbs 1909, Campbell 1971, Hürlimann 1989.

** — var. striatus OV: Miller et al. 1983. VI: Miller et al. 1983.

\section{Pycnolejeunea}

* ${ }^{17}$ P. convexifolia (Mitt.) Steph. FIJ: TYPE of Lejeunea convexifolia, Mitten 1873 as Lejeunea convexifolia, Stephani 1897 as Pycnolejeunea convexistipa, 1914, Miller et al. 1983 also as Pycnolejeunea convexistipa and Lejeunea convexifolia, Geissler \& Bischler 1987 as Lejeunea convexifolia. VI: He 1999.

${ }^{15}$ Plicanthus birmensis seems to be close to Tetralophozia (N. Konstantinova, pers. comm. 2009).

${ }^{16}$ Preliminary investigation of New Zealand material suggests that Porella elegantula is a complex of three forms. The type of P. elegantula is from Auckland Islands, and appears to be more or less confined to western and southern South Island, and the subantartics, and seems to be endemic to New Zealand. The record here is thus doubtful and needs clarification.

${ }^{17}$ Pycnolejeunea convexifolia may be a form of Cheilolejeunea trapezia (X.-L.He, pers. comm.). 


\section{Radula}

* ${ }^{18}$ R. acuminata Steph. FIJ: Yamada \& Piippo 1989, Zhu \& So 2001, So 2006. TA: Pócs et al. 2011. VI: Yamada 1984.

** R. acuta Mitt. TA: So 2006.

*** R. amentulosa Mitt. FII: TYPE, Mitten 1873, Stephani 1910, Herzog 1953, Kitagawa 1973b, Yamada 1975, 1979, Thiers 1983, Miller et al. 1983, Yamada 1985, 1999, Yamada \& Piippo 1989, Geissler \& Bischler 1990. OV: So 2006. TA: So 2006. VA: So 2006. VI: Mitten 1861, Yamada 1984, So 2006.

*** R. campanigera Mont. OV: Campbell 1971, Miller et al. 1983. TA: Campbell 1971, Miller et al. 1983. VI: Campbell 1971, Miller et al. 1983.

*** R. decurrens Mitt. OV: Castle 1965.

$\star * * \quad$ R. formosa (C.F.W.Meissn. ex Spreng.) Nees FII: TYPE of Radula spicata, Mitten 1862, 1873 both as Radula spicata, Stephani 1910, Castle 1950, Inoue \& Miller 1965, Grolle 1966, Kitagawa 1979, Yamada 1979, 1999, Thiers 1983 as Radula spicata, Miller et al. 1983 as Radula spicata, Yamada \& Piippo 1989, Geissler \& Bischler 1990 as Radula spicata, So 2006. KA: So 2006. VA: Campbell 1971, Miller et al. 1983. VI: Hürlimann 1978, Miller et al. 1983, So 2006.

*** $\quad$ R. javanica Gottsche OV: Castle 1966. TA: So 2006. VI: So 2006.

*** R. lingulata Gottsche FII: So 2006. VI: Yamada 1984.

* ${ }^{19}$ R. multiflora Gottsche ex Schiffn. KA: Pócs et al. 2011. TA: Pócs et al. 2011. VI: Yamada 1984.

* ${ }^{20}$ R. nymannii Steph. FIJ: Yamada 1979, Miller et al. 1983, Yamada \& Piippo 1989. $\underline{\mathrm{TA}}$ : current report. $\underline{\mathrm{VI}}$ : current report.

*** R. protensa Lindenb. TA: Pócs et al. 2011.

** — var. erectilobula Schiffn. VI: Campbell 1971 ["errectilobula"], Miller et al. 1983.

*** $\quad R$. reflexa Nees et Mont. TA: So 2006. VI: So 2006.

$\star * * \quad$ R. retroflexa Taylor TA: So 2006. VA: Campbell 1971 as Radula miqueliana, Miller et al. 1983 as Radula retroflexa var. retroflexa. VI: Campbell 1971 as Radula miqueliana, Miller et al. 1983 as Radula retroflexa var. retroflexa.

*** R. scariosa Mitt. FII: TYPE, Mitten 1873, Stephani 1910, Castle 1950, Thiers 1983, Miller et al. 1983, Yamada 1985 as Radula scariosa var. scariosa, 1999, Geissler \& Bischler 1990. VI: Mitten 1861, So 2006.

*** R. tjibodensis K.I.Goebel FIJ: Zhu \& So 2001, Eggers \& Pócs 2010. TA: Campbell 1971, Miller et al. 1983. VI: Campbell 1971, Hürlimann 1978, Miller et al. 1983, So 2006.

${ }^{18}$ Radula acuminata is very close to $R$. tjibodensis (Söderström et al. 2010).

${ }_{19}$ Radula multiflora was treated as a new synonym of Radula javanica by So (2006), but accepted by Renner and de Lange (2009), who record this species as new for New Zealand, where $R$. javanica also occurs.

${ }^{20}$ Radula nymannii is close to $R$. protensa (Söderström et al. 2010). We have not found any first hand report of it from Fiji in the literature although Yamada (1979) reports it but we are able to cite the following specimens: Taveuni, Wainibau Stream, leg. S \& T Pócs 03826/ AR; road to De Voeux Peak, leg. S. \& T. Pócs 03288/DP; Viti Levu, South Coast, S \& T Pócs 03261/CA, all det. M. Renner, 2011. 


\section{Riccardia}

*** R. alba (Colenso) E.A.Br. FII: Miller et al. 1983 as Riccardia vitiensis, Geissler \& Bischler 1990 as Riccardia vitiensis. OV: TYPE of Aneura samoana and Aneura vitiensis, Stephani 1893a as Aneura vitiensis and Aneura samoana ["SamoaOvalau”], 1899c as Aneura vitiensis, Jack \& Stephani 1894 as Aneura vitiensis, Bonner 1962b as Aneura vitiensis, Campbell 1971 as Riccardia vitiensis, Miller et al. 1983 as Riccardia samoana, Aneura samoana and Aneura vitiensis, Brown \& Braggins 1989. VA: Campbell 1971 as Riccardia vitiensis, Miller et al. 1983 as Riccardia samoana. VI: Campbell 1971 as Riccardia vitiensis, Hürlimann 1976 as Riccardia samoana, Miller et al. 1983 as Riccardia samoana.

** R. flagellaris (A.Gepp) H.A.Mill. FIJ: Schuster 1992a as Aneura flagellaris. VI: TYPE of Aneura flagellaris, Gibbs 1909 as Aneura flagellaris, Bonner 1977 as Aneura flagellaris, Miller et al. 1983 also as Aneura flagellaris.

*** R. graeffei (Steph.) Hewson FII: Geissler \& Bischler 1990, Furuki 2002, Furuki 2006. VI: TYPE of Aneura graeffei, Stephani 1893a as Aneura graeffei, Jack \& Stephani 1894 as Aneura graeffei, Bonner 1962b as Aneura graeffei, Hürlimann 1976, Miller et al. 1983 also as Aneura graeffei, Furuki 1991.

** R. heteroclada Schiffn. TA: Campbell 1971 as Riccardia serrulata, Miller et al. 1983 as Riccardia serrulata.

** R. nadeaudii (Steph.) Hürl. VI: Gibbs 1909 as Aneura nadeaudii, Miller et al. 1983.

** $\quad R$. pectinata (Steph.) Hürl. var. fasciculata FII: Miller et al. 1983 as Riccardia fasciculata. VI: Hürlimann 1976, Miller et al. 1983.

** R. plumosa (Mitt.) E.O.Campb. FII: TYPE of Sarcomitrium plumosum, Mitten 1862, 1873 both as Sarcomitrium plumosum, Stephani 1899b as Aneura plumosa, Bonner 1962b as Aneura plumosa, Thiers 1983 as Sarcomitrium plumosum, Miller et al. 1983 as Sarcomitrium plumosum and Aneura plumosa, Geissler \& Bischler 1990 also as Sarcomitrium plumosum. VI: Campbell 1971, Hürlimann 1976, Miller et al. 1983.

** R. tenuicostata Schiffn. VI: Gibbs 1909 as Aneura tenuicostata, Miller et al. 1983.

\section{Riccia}

** R. linearis (Schiffn.) Steph. FII: Stephani 1898, Geissler \& Bischler 1990. VI: TYPE of Ricciella linearis, Schiffner 1890 as Ricciella linearis, Miller et al. 1983, Geissler \& Bischler 1990 as Ricciella linearis.

** $\quad$ R. rechingeri Steph. VI: Campbell 1971, Miller et al. 1983.

\section{Saccogynidium}

** S. chiloscyphoides R.M.Schust. VA: TYPE, Schuster 1963, Miller et al. 1983, Geissler \& Bischler 1990.

** S. muricellum (De Not.) Grolle FIJ: Grolle \& Schultze-Motel 1973, Miller et al. 1983, Piippo 1985, Hürlimann 1998, Gao et al. 2001.

** S. rigidulum (Nees) Grolle FIJ: Grolle 1965, 1968 both also as Saccogynidium jugatum, Grolle \& Schultze-Motel 1973, Miller et al. 1983 as Saccogynidium jugatum, Piippo 1985, Gao et al. 2001. VA: Miller et al. 1983. 


\section{Sandeothallus}

** S. radiculosus (Schiffn.) R.M.Schust. VI: Campbell 1971 as Calycularia radiculosa, Miller et al. 1983 as Moerckia radiculosa.

\section{Schistochila}

*** S. aligera (Nees et Blume) J.B.Jack et Steph. FII: Miller et al. 1983 as Paraschistochila graeffeana, Geissler \& Bischler 1989 as Paraschistochila graeffeana, 1990 as Schistochilaster graeffeanus. OV: TYPE of Schistochila graeffeana, Jack \& Stephani 1894 as Schistochila graeffeana, Stephani 1909 as Schistochila graeffeana, Miller et al. 1983 as Schistochilaster graeffeanus and Schistochila graeffeana, Geissler \& Bischler 1990 as Schistochila graeffeana. VA: Campbell 1971 as Schistochila philippinensis, Miller et al. 1983 as Schistochilaster philippinensis. VI: Campbell 1971 as Schistochila philippinensis, Miller et al. 1983 as Schistochilaster philippinensis.

*** S. blumei (Nees) Trevis. VI: Hürlimann 1998.

$\star * * \quad$ S. caledonica Steph. FII: Hürlimann 1998. VI: Campbell 1971 as Schistochila flavicans, Miller et al. 1983.

*** S. fijiensis H.Buch et Herzog TA: Campbell 1971, Miller et al. 1983. VA: TYPE, Herzog 1951a, Campbell 1971, Miller et al. 1983, Geissler \& Bischler 1990. VI: Campbell 1971, Miller et al. 1983.

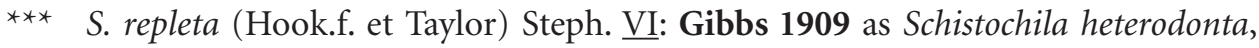
Miller et al. 1983 as Schistochila heterodonta.

*** S. sciurea (Nees) Schiffn. FII: Schiffner 1898. OV: Jack \& Stephani 1894, Campbell 1971, Miller et al. 1983 also as Ptilidium sciureum and Schistocheila sciurea. VI: Miller et al. 1983 as Gottschea sciurea and Jungermannia sciurea, Hürlimann 1998.

\section{Solenostoma}

*** S. ariadne (Taylor ex Lehm.) R.M.Schust. ex Váňa et D.G.Long FII: Váňa 1973a, 1975, 1991a all as Jungermannia ariadne, Váňa \& Piippo 1989a as Jungermannia ariadne, Bapna \& Kachroo 2000 as Jungermannia ariadne, Schuster 2002 as Solenostoma ariadne. VA: Váňa 1973b as Jungermannia ariadne, Miller et al. 1983 as Plectocolea ariadne. VI: Váňa $\mathbf{1 9 7 3 b}$ as Jungermannia ariadne, Miller et al. 1983 as Plectocolea ariadne.

*** S. haskarlianum (Nees) R.M.Schust. ex Váňa et D.G.Long FII: Váňa 1973a, 1991a both as Jungermannia haskarliana, Váňa \& Inoue 1983 as Jungermannia haskarliana, Váňa \& Piippo 1989a as Jungermannia haskarliana, Bapna \& Kachroo 2000 as Jungermannia haskarliana, Gao \& Bai 2001 as Jungermannia haskarliana, Schuster 2002 as Solenostoma hasskarlianum. VA: Campbell 1971 as Plectocolea haskarliana. VI: Campbell 1971 as Plectocolea haskarliana, Váňa 1973b, 1975 both as Jungermannia haskarliana, Hürlimann 1974 as Jungermannia haskarliana ["hasskarliana"], Miller et al. 1983 as Jungermannia haskarliana.

*** S. tetragonum (Lindenb.) R.M.Schust. ex Váňa et D.G.Long FII: Váňa \& Piippo 1989a as Jungermannia tetragona, Váňa 1991a as Jungermannia tetragona, Schuster 2002 as Solenostoma tetragonum, Váňa et al. 2005 as Jungermannia tetragona. TA: Campbell 1971 as Plectocolea tetragona, Váňa 1975 as Jungermannia tetragona, Miller et al. 1983 as Jungermannia tetragona. VA: Campbell 1971 as Plectocolea tetragona, Miller et al. 1983 as Jungermannia tetragona. VI: Campbell 1971 as Plectocolea tetragona, Miller et al. 1983 as Jungermannia tetragona. 
*** S. truncatum (Nees) R.M.Schust. ex Váňa et D.G.Long FII: Váňa 1991a as Jungermannia truncata, Piippo et al. 1998 as Jungermannia truncata, Váňa et al. 2005 as Jungermannia truncata. VI: Váňa \& Piippo 1989a as Jungermannia truncata.

\section{Spruceanthus}

*** S. polymorphus (Sande Lac.) Verd. KA: Pócs et al. 2011. OV: Campbell 1971, Miller et al. 1983. VI: Campbell 1971, Miller et al. 1983.

\section{Symphyogynopsis}

*** S. gottscheana (Mont. et Nees) Grolle FIJ: Furuki 2002 as Symphyogynopsis filicum, Chantanaorrapint et al. 2004 as Symphyogynopsis filicum. OV: TYPE of Symphyogyna vitiensis, Jack \& Stephani 1894 as Symphyogyna vitiensis, Stephani 1900 as Symphyogyna vitiensis, Miller et al. 1983 as Symphyogyna vitiensis, Grolle \& Piippo 1986 as Symphyogynopsis filicum, Geissler \& Bischler 1990 as Symphyogyna vitiensis. VI: Gibbs 1909 as Symphyogyna vitiensis, Hürlimann 1969 as Symphyogyna vitiensis, Campbell 1971 as Symphyogyna vitiensis, Miller et al. 1983 as Symphyogyna vitiensis, Grolle \& Piippo 1986 as Symphyogynopsis filicum.

\section{Taxilejeunea}

** T. deflexa Steph. FIJ: TYPE, Stephani 1914, Eifrig 1937, Geissler \& Bischler 1990. VI: Miller et al. 1983.

\section{Telaranea}

*** T. lindenbergii (Gottsche) J.J.Engel et G.L.Merr. VI: Gibbs 1909 as Lepidozia lindenbergii.

*** T. pruinosa (Herzog) J.J.Engel et G.L.Merr. OV: TYPE of Lepidozia pruinosa, Herzog 1951a as Lepidozia pruinosa, Miller et al. 1983 as Lepidozia pruinosa, Geissler \& Bischler 1985 as Lepidozia pruinosa, Engel \& Smith Merrill 2004.

*** T. rosarioana H.A.Mill. FIJ: Engel \& Smith Merrill 2004 [the locality is in Vanuatu, not in Fiji]. VI: Pócs et al. 2011.

\section{Temnoma}

*** T. setigerum (Lindenb.) R.M.Schust. FIJ: Schuster 1969, Piippo 1984a, Schuster 1984, Long \& Grolle 1990, Bapna \& Kachroo 2000, Schuster 2000. VI: Schuster 1968.

** 21 — var. hawaiicum Inoue FII: Miller et al. 1983.

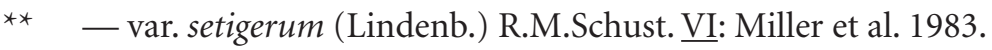

\section{Thysananthus}

*** T. retusus (Reinw., Blume et Nees) B.M.Thiers et Gradst. FII: Mitten 1873 as Phragmicoma plana, Schiffner 1898 as Thysananthus planus, Miller et al. 1983 as

${ }^{21}$ Temnoma setigerum is reported from Fiji both as var. setigerum and var. hawaiicum by Miller et al. (1983), but we are not aware of any first hand report of any of the varieties and do not know what they are basing their reports on. 
Phragmicoma plana. VA: Verdoorn 1937 as Thysananthus planus, Campbell 1971 as Thysananthus planus, Miller et al. 1983 as Thysananthus planus. VI: Campbell 1971 as Caudalejeunea stephanii, Miller et al. 1983 as Thysananthus planus, Hürlimann 1989 as Thysananthus planus.

*** T. spathulistipus (Reinw., Blume et Nees) Lindenb. KA: Pócs et al. 2011. OV: Jack \& Stephani 1894 as Thysanolejeunea spathulistipa, Campbell 1971, Miller et al. 1983. VI: Campbell 1971, Hürlimann 1989.

\section{Treubia}

** T. insignis (Steph.) R.M.Schust. et G.A.M.Scott. FIJ: Piippo 1988.

** - subsp. bracteata (Steph.) R.M.Schust. et G.A.M.Scott FIJ: Schuster 1963 as Treubia bracteata. TA: Campbell 1971 as Treubia bracteata, Miller et al. 1983. VI: Gibbs 1909 as Treubia bracteata, Miller et al. 1983.

** - subsp. vitiensis R.M.Schust. et G.A.M.Scott FIJ: Schuster \& Konstantinova 1995. VI: TYPE, Schuster \& Scott 1969, Miller et al. 1983, Geissler \& Bischler 1990.

*** T. lacunosa (Colenso) Prosk. VI: Gibbs 1909 as Treubia bracteata.

\section{Trichocolea}

* ${ }^{22}$ T. pluma (Reinw., Blume et Nees) Mont. FIJ: Grolle 1965, Kitagawa 1973b, Kitagawa 1978. OV: Jack \& Stephani 1894, Miller et al. 1983. TA: Campbell 1971. VA: Campbell 1971. VI: Gibbs 1909, Campbell 1971, Hürlimann 1983, Miller et al. 1983.

*** T. tomentella (Ehrh.) Dumort. FIJ: Mitten 1861.

\section{Zoopsidella}

*** Z. caledonica (Steph.) R.M.Schust. FIJ: Schuster 1969, Grolle \& Piippo 1984, Schuster 2000. VI: Schuster 1970, Schuster 1999.

\section{Zoopsis}

** Z. liukiuensis Horik. VA: Pócs et al. 2011. VI: Pócs et al. 2011.

\section{Taxa doubtfully occurring on Fiji}

List of taxa reported from Fiji that we regard questionable and in need of verification either from re-examination of specimens or new reports.

\section{Frullania}

* ${ }^{23}$ F. brotheri Steph. FI]: Stephani 1911, Bonner 1965. VI: Miller et al. 1983 [Viti Levu with a "?"].

22 Trichocolea pluma is almost inseparable from T. tomentella and often included in the latter (Söderström et al. 2010).

${ }^{23}$ We know of no first hand report of Frullania brotheri from Fiji. Furthermore, it is taxonomically questionable and possibly just a form of $F$. nodulosa (Söderström et al. 2010). 
** 24 F. hamata Steph. FII: Pócs 2008b. VI: Campbell 1971, Miller et al. 1983.

$\star * \star 25$ F. hypoleuca Nees FIJ: von Konrat \& Braggins 2001, Pócs 2008b. OV: Miller et al. 1983.

\section{Heteroscyphus}

** ${ }^{26}$ H. caledonicus (Steph.) Schiffn. FII: Miller et al. 1983, Hürlimann 1998.

** ${ }^{27}$ H. parvulus (Schiffn.) Schiffn. VI: Gibbs 1909 as Chiloscyphus parvulus.

\section{Lejeunea}

** ${ }^{28}$ L. papilionacea Steph. Fij: Bonner 1966 as Hygrolejeunea diversitexta.

\section{Schiffneriolejeunea}

$\star * \star 29$ S. tumida (Gottsche) Gradst. et Terken var. haskarliana VA: Miller et al. 1983 as Schiffneriolejeunea haskarliana. VI: Miller et al. 1983 as Schiffneriolejeunea haskarliana.

\section{Telaranea}

***30 T. wallichiana (Gottsche) R.M.Schust. VI: Campbell 1971 as Lepidozia tenera, Miller et al. 1983 as Lepidozia tenera.

${ }^{24}$ Frullania hamata is given as endemic to New Guinea by Hattori \& Piippo (1986) without commenting on the records from Fiji. We treat the report from Fiji as doubtful until the identity of the specimen is confirmed.

${ }^{25}$ We know of no first hand report of Frullania hypoleuca from Fiji and we do not know what Miller et al. (1983) are basing their report on. However, they include taxa in their synonym list that we refer elsewhere and it may be some of these, but $F$. hypoleuca may well occur in Fiji since it occurs on other islands in the Pacific.

${ }^{26}$ We know of no first hand reports of Heteroscyphus caledonicus and none of the references Miller et al. (1983) cite seem to mention it for Fiji.

${ }^{27}$ Heteroscyphus parvulus occurs only in Java, Borneo and New Guinea according to Piippo (1992). We treat the report from Fiji as doubtful unit the identity of the specimen confirmed.

28 The only report of Lejeunea papilionaceae from the Pacific is a second hand report by Bonner (1966) from Fiji and New Caledonia. It is a taxon from Africa and SE Asia.

29 We know of no first hand reports of Schiffneriolejeunea tumida from Fiji and do not know what Miller et al. (1983) are basing their reports on. However, it is widely distributed in the Pacific so it probably also occurs in Fiji.

30 Telaranea wallichiana is a species complex that was treated by Engel \& Smith Merrill (2004), but they do not report it from anywhere in the Pacific although earlier reports exist. 


\section{Taxa rejected from Fiji}

List of taxa reported from Fiji that has been shown erroneous or are here rejected.

\section{Cheilolejeunea}

***31 C. mariana (Gottsche) B.M.Thiers et Gradst. FIJ: Stephani 1911 as Archilejeunea mariana. VA: Campbell 1971 as Archilejeunea mariana, Miller et al. 1983 as Spruceanthus marianus. VI: Campbell 1971 as Archilejeunea mariana, Miller et al. 1983 as Spruceanthus marianus.

\section{Frullania}

***32 F. orientalis Sande Lac. VI: Campbell 1971, Miller et al. 1983.

\section{Hygrolejeunea}

* ${ }^{33}$ H. parvicalycina Steph. FIJ: Bonner 1966, Miller et al. 1983.

\section{Lejeunea}

** ${ }^{34}$ L. tenella Taylor VI: Campbell 1971 as Euosmolejeunea tenella, Miller et al. 1983 as Euosmolejeunea tenella.

\section{Lepicolea}

${ }^{* * *}{ }^{35}$ L. scolopendra (Hook.) Dumort. ex Trevis. FIJ: Fulford 1963, Hässel \& Solari 1975, Miller et al. 1983, Solari 1983, Schuster 2000.

\section{Lepidolaena}

** ${ }^{36}$ L. brachyclada (Taylor ex Lehm.) Trevis. FIJ: Miller et al. 1983 [with a “?”].

\section{Leptolejeunea}

** ${ }^{37}$ L. lancifolia (Mitt.) Steph. FIJ: Miller et al. 1983 [with a “?”].

31 Thiers \& Gradstein (1989) showed that most of the reports of Cheilolejeunea mariana belongs to Archilejeunea planiuscula. C. mariana is confined to the Mariana Is. and Caroline Is.

32 All reports of Frullania orientalis from Fiji are Frullania capillaris (Hattori 1985).

33 The genus Hygrolejeunea is a now generally regarded as a synonym of Lejeunea but $H$. parvicalycina has never been transferred and we do not know the value of it. Furthermore, we know of no first hand report and can not find what Bonner (1965) is basing his report on. It is otherwise confined to Java.

34 Lejeunea tenella is rejected from the Pacific by Zhu (2006).

35 Lepicolea scolopendra is a taxon from Australia and New Zealand erronously reported from many parts of the world.

36 Lepidolaena brachyclada is endemic to Tasmania (Schuster 1984).

37 Leptolejeunea lancifolia is reported from Fiji by Miller et al. (1983) probably because it was published in Flora Vitiensis by Mitten (1873), but he clearly states that it is from Samoa. Mitten reported many new things from areas other than Fiji in that publication, and several authors have been too uncritical and reported many taxa from Fiji that never have been found there, because of this unfortunate way of publishing. 


\section{Lopholejeunea}

$* * * 38$ L. plicatiscypha (Hook.f. et Taylor) Steph. FII: Stephani 1912 as Lopholejeunea tecta, Miller et al. 1983 [with a “?”], Thiers \& Gradstein 1989.

\section{Marchantia}

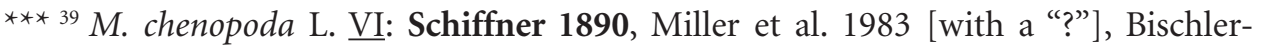
Causse 1989.

** ${ }^{40}$ M. paleacea Bertol. FI]: Mitten 1873 [s.loc.spec.], Evans 1917 [with doubt], Hattori 1966 [with a “?”], Hattori 1968 [with a “?”], Bapna \& Kachroo 2000b. OV: Miller et al. 1983 [with a “?”]. VI: Miller et al. 1983 [with a “?”].

*** — subsp. paleacea FII: Schiffner 1898 as Marchantia nitida, Miller et al. 1983 as Marchantia nitida, Schuster 1992b [with a “?”]. OV: Jack \& Stephani 1894 as Marchantia nitida. VI: Mitten 1873 as Marchantia nitida.

${ }^{\star * \star}{ }^{41}$ M. pileata Mitt. FII: Mitten 1862, Miller et al. 1983.

\section{Mastigolejeunea}

*** ${ }^{42}$ M. virens (Ångstr.) Steph. FIJ: Miller et al. 1983 as Mastigolejeunea taitica.

\section{Metzgeria}

${ }^{* * *}{ }^{43}$ M. procera Mitt. VI: Kuwahara 1966 as Metzgeria hamata, Campbell 1971 as Metzgeria hamata.

\section{Plagiochila}

*** ${ }^{44}$ P. blepharophora (Nees) Lindenb. FIJ: Miller et al. 1983.

\section{Plagiochilion}

** ${ }^{45}$ P. combinatum (Mitt.) Inoue FII: Stephani 1904 b as Plagiochila combinata, Miller et al. 1983 also as Plagiochila combinata [both with a "?"].

${ }^{38}$ Lopholejeunea plicatiscypha is reported from Fiji by Stephani (1912; as Lopholejeunea tecta) probably because it was published in Flora Vitiensis by Mitten (1873), but there he clearly states that it is from Kermadec. Mitten reported many new things from areas other than Fiji in that publication, and several authors have been too uncritical and reported many taxa from Fiji that never have been found there, because of this unfortunate way of publishing.

${ }^{39}$ Marchantia chenopoda is an American species (Bischler-Causse 1989).

${ }^{40}$ All reports of Marchantia paleacea refer to M. vitiensis (Bischler-Causse 1989).

${ }^{41}$ All reports of Marchantia pileata refer to M. vitiensis (Bischler-Causse 1989).

${ }^{42}$ Mastigolejeunea virens is reported from Fiji based on complicated synonymy. We know of no reliable report although it occurs on many islands in the South Pacific.

${ }^{43}$ Metzgeria procera is an American species erroneously reported from many parts of the world due to complicated synonymy.

${ }^{44}$ Plagiochila blepharophora is reported due to erronous synonymy with $P$. vitiensis. 


\section{Porella}

** ${ }^{46}$ P. javanica (Gottsche ex Steph.) Inoue VI: Campbell 1971, Miller et al. 1983.

\section{Psiloclada}

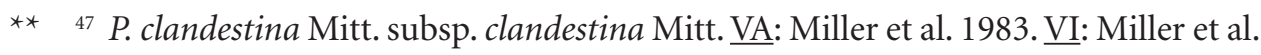
1983.

\section{Radula}

** ${ }^{48}$ R. anceps Sande Lac. FII: So 2006. VI: Campbell 1971, Miller et al. 1983 as Radula anceps var. anceps.

${ }^{* * *}{ }^{49}$ R. brunnea Steph. FIJ: Miller et al. 1983.

*** ${ }^{50}$ R. complanata (L.) Dumort. OV: Miller et al. 1983.

\section{Riccardia}

${ }^{\star * *}{ }^{51}$ R. chamedryfolia (With.) Grolle VI: Schiffner 1890 as Aneura pinnatifida.

\section{Symbiezidium}

*** 52 S. transversale (Sw.) Trevis. var. transversale FII: Verdoorn 1934 as Symbiezidium bacciferum, Miller et al. 1983 as Symbiezidium bacciferum.

\section{Telaranea}

*** ${ }^{53}$ T. bisetula (Steph.) E.O.Campb. FIJ: Engel \& Smith Merrill 2004. VI: Campbell 1971, Miller et al. 1983 also as Lepidozia bisetula.

*** 54 T. melanesica H.A.Mill. FII: Engel \& Smith Merrill 2004.

*** ${ }^{5}$ T. tetradactyla (Hook.f. et Taylor) E.A.Hodgs. VI: Váňa 1975 as Jungermannia tetradactyla, Miller et al. 1983, Hürlimann 1985, Engel \& Smith Merrill 2004.

${ }^{45}$ We know of no reliable report of Plagiochilion combiantum outside Hawaii.

${ }^{46}$ Hattori (1985) doubted the report of Porella javanica from Fiji but did not study any specimen and So (2002) rejected it after seeing the specimens the report was based on.

${ }^{47}$ All reports of Psiloclada clandestina from Fiji should be referred to subsp. melanesica.

${ }^{48}$ Radula anceps was rejected from Fiji by So (2006).

${ }^{49}$ Kitagawa (1973b) rejected earlier reports of Radula brunnea from Fiji as a result of the erronous synonymization with $R$. amentulosa by Castle (1950).

${ }^{50}$ Radula complanata is a boreal taxon that almost certainly does not occur in Fiji. We have not found the source for the report in Miller et al. (1983).

${ }^{51}$ Riccardia chamedryfolia is a boreal species not occurring in Fiji.

52 Symbiezidium transversale is an American species (Gradstein \& van Beek 1975).

${ }^{53}$ The report of Telaranea bisetula by Campbell (1971) is rejected by Hürlimann (1985).

${ }_{54}$ The locality for Telaranea melanesica is in Vanuatu, not in Fiji.

${ }^{55}$ Engel \& Smith Merrill (2004) reject all earlier reports of Telaranea tetradactyla from Fiji. 


\section{Treubia}

** ${ }^{56}$ T. insignis K.I.Goebel subsp. insignis VI: Miller et al. 1983.

\section{Tylimanthus}

*** 57 T. saccatus (Hook.) Mitt. ex Carrington et Pearson FIJ: Mitten 1873 as Marsupidium urvilleanum, Miller et al. 1983 as Tylimanthus urvilleanus [with a "?"], Gymnanthe urvilleana and Marsupidium urvilleanum.

\section{Synonyms}

Only synonyms used in reports from Fiji are included.

Anastrophyllum contractum (Reinw., Blume et Nees) Steph. $\equiv$ Cuspidatula contracta

Anastrophyllum graeffei J.B.Jack et Steph. = Denotarisia linguifolia

Anastrophyllum vitiense J.B.Jack et Steph. = Cuspidatula contracta

Aneura flagellaris A.Gepp $\equiv$ Riccardia flagellaris

Aneura graeffei Steph. $\equiv$ Riccardia graeffei

Aneura lobata (Schiffn.) Steph. = Lobatiriccardia coronopus

Aneura nadeaudii Steph. $\equiv$ Riccardia nadeaudii

Aneura pinnatifida (Weber) Nees = Riccardia chamedryfolia

Aneura plumosa (Mitt.) Steph. $\equiv$ Riccardia plumosa

Aneura samoana Steph. $=$ Riccardia alba

Aneura tenuicostata (Schiffn.) Steph. $\equiv$ Riccardia tenuicostata

Aneura vitiensis Steph. $=$ Riccardia alba

Anthoceros amboinensis Schiffn. $\equiv$ Folioceros amboinensis

Anthoceros appendiculatus Steph. = Folioceros glandulosus

Anthoceros flagellaris Mitt. $\equiv$ Megaceros flagellaris

Anthoceros fuciformis Mont. $\equiv$ Folioceros fuciformis

Anthoceros glandulosus Lehm. et Lindenb. $\equiv$ Folioceros glandulosus

Anthoceros laevis subsp. carolinianus (Michx.) Prosk. $\equiv$ Phaeoceros carolinianus

Archilejeunea calcarata (Mont.) Mitt. ex Steph. = Mastigolejeunea calcarata

Archilejeunea graeffei J.B.Jack et Steph. $\equiv$ Lepidolejeunea graeffei

Archilejeunea mariana (Gottsche) Steph. $\equiv$ Cheilolejeunea mariana

Archilejeunea meyeniana (Nees, Lindenb. et Gottsche) J.B.Jack et Steph. $\equiv$ Cheilolejeunea meyeniana

${ }^{56}$ Treubia insignis subsp. insignis is a SE Asiatic taxon and only subsp. bracteata and vitiensis occurs in Fiji.

${ }^{57}$ All reports of Tylimanthus saccatus originate from Mitten (1871) where he reports it as Marsupidium urvilleanum saying "A single stem picked from amongst Mosses is all that indicates the presence of this or some closely allied species". We reject the report due to this vague statement, and the fact that the species is not reported from anywhere else in the Pacific. 
Aspiromitus glandulosus (Lehm. et Lindenb.) Steph. $\equiv$ Folioceros glandulosus

Bazzania australis (Mont.) Trevis. = Bazzania tridens

Bazzania australis Gottsche, Lindenb. et Nees = Bazzania tridens

Bazzania vitiensis Mitt. ex E.O.Campb. nom. inval. = Bazzania vitiana

Bryopteris sinclairii (Mitt.) Mitt. $\equiv$ Lejeunea sinclairii

Bryopteris striata (Lehm. et Lindenb.) Mitt. $\equiv$ Ptychanthus striatus

Calycularia radiculosa Steph. ex Schiffn. nom. illeg. = Sandeothallus radiculosus

Caudalejeunea recurvistipula (Gottsche) Schiffn. = Caudalejeunea reniloba

Caudalejeunea stephanii Spruce ex Steph. = Thysananthus retusus

Chandonanthus birmensis Steph. $\equiv$ Plicanthus birmensis

Chandonanthus hirtellus (F.Weber) Mitt. $\equiv$ Plicanthus hirtellus

Cheilolejeunea parkinsonii (Steph.) Mizut. = Cheilolejeunea cookiensis

Chiloscyphus argutus (Reinw., Blume et Nees) Nees $\equiv$ Heteroscyphus argutus

Chiloscyphus aselliformis (Reinw., Blume et Nees) Nees $\equiv$ Heteroscyphus aselliformis

Chiloscyphus communis Steph. = Heteroscyphus coalitus

Chiloscyphus confluens Mitt. = Heteroscyphus aselliformis

Chiloscyphus endlicherianus (Nees) Nees = Heteroscyphus argutus var. argutus

Chiloscyphus parvulus Schiffn. $\equiv$ Heteroscyphus parvulus

Chiloscyphus succulentus Gottsche $\equiv$ Heteroscyphus succulentus

Cololejeunea goebelii (Gottsche ex Schiffn.) Schiffn. = Cololejeunea trichomanis

Cololejeunea hispidissima (Steph.) Pandé, K.P.Srivast. et Sultan Khan = Cololejeunea haskarliana var. haskarliana

Cololejeunea nymannii (Steph.) Benedix = Cololejeunea obliqua

Cololejeunea vitiensis (E.O.Campb.) H.A.Mill. nom. illeg. = Cololejeunea polyantha

Colura acutifolia Ast = Colura conica

Colura apiculata Schiffn. ex Steph. = Colura leratii

Colurolejeunea apiculata Schiffn. nom. inval. = Colura leratii

Crossogyna flexicaulis (Nees) Schljakov $\equiv$ Jamesoniella flexicaulis

Cuspidatula vitiensis (J.B.Jack et Steph.) Steph. = Cuspidatula contracta

Dendroceros pusillus Steph. $=$ Dendroceros javanicus

Dendrolejeunea vittata (Mitt.) Steph. ex Schiffn. nom. illeg. = Dendrolejeunea fruticosa

Diplasiolejeunea brachyclada A.Evans = Diplasiolejeunea cavifolia

Diplomitrion lyellii (Hook.) Corda $\equiv$ Pallavicinia lyellii

Drepanolejeunea filicuspis Steph. $\equiv$ Harpalejeunea filicuspis

Drepanolejeunea riddleana Steph. = Harpalejeunea filicuspis

Drepanolejeunea ternatensis var. lancispina Herzog = Drepanolejeunea ternatensis

Drepanolejeunea uncinata (Mitt.) Steph. = Drepanolejeunea ternatensis

Dumortiera trichocephala (Hook.) Nees = Dumortiera hirsuta subsp. nepalensis

Dumortiera velutina Schiffn. $=$ Dumortiera hirsuta subsp. nepalensis

Euosmolejeunea parkinsonii Steph. = Cheilolejeunea cookiensis

Euosmolejeunea partiicola (Reichardt) J.B.Jack et Steph. = Cheilolejeunea trifaria 
Euosmolejeunea tenella (Taylor) Steph. $\equiv$ Lejeunea tenella

Euosmolejeunea trifaria (Reinw., Blume et Nees) Spruce ex Schiffn. $\equiv$ Cheilolejeunea trifaria Euosmolejeunea uvifera (Mont.) Steph. = Lejeunea flava

Folioceros spinisporus (Steph.) D.C.Bharadwaj = Folioceros amboinensis

Frullania angulistipa (Steph.) Steph. nom. inval. $\equiv$ Frullania angustistipa

Frullania angulosa Mitt. = Frullania intermedia subsp. intermedia

Frullania curvatirostra J.B.Jack ex Steph. nom. inval. = Frullania novocurvirostris

Frullania curvirostris J.B.Jack et Steph. nom. illeg. 三 Frullania novocurvirostris

Frullania grossispica Steph. = Frullania serrata

Frullania inflatistipula J.B.Jack ex Bonner nom. inval. = Frullania nodulosa

Frullania intermedia f. billardieriana (Nees et Mont.) Verd. = Frullania intermedia subsp. intermedia

Frullania khedingiana Steph. = Frullania intermedia subsp. intermedia

Frullania meteoroides Mitt. = Frullania serrata

Frullania pacificae Taylor $=$ Frullania apiculata

Frullania parhamii (R.M.Schust.) R.M.Schust. nom. inval. $\equiv$ Frullania parhamii

Frullania pendula Austin nom. illeg. = Frullania deflexa

Frullania rechingeri Steph. $=$ Frullania immersa

Frullania squarrosa $($ Nees) Nees = Frullania ericoides

Frullania stellatitexta Steph. = Frullania intermedia subsp. intermedia

Frullania varians J.B.Jack ex Bonner nom. inval. = Frullania deflexa

Frullania vesiculosa Steph. $=$ Frullania ericoides

Frullania vethii Sande Lac. $=$ Frullania trichodes

Gottschea sciurea (Nees) Sande Lac. $\equiv$ Schistochila sciurea

Gymnanthe urvilleana (Mont.) Taylor = Tylimanthus saccatus

Harpalejeunea owaihiensis (Gottsche) A.Evans = Lepidolejeunea involuta

Herbertus tutuilanus Pearson $=$ Herbertus longifissus

Herpetium australe Mont. $=$ Bazzania tridens

Heteroscyphus communis (Steph.) Schiffn. = Heteroscyphus coalitus

Heteroscyphus concinnus (De Not.) Schiffn. = Heteroscyphus succulentus

Heteroscyphus confluens (Mitt.) Schiffn. = Heteroscyphus aselliformis

Hygrolejeunea decurvifolia Steph. = Lepidolejeunea bidentula

Hygrolejeunea devexiloba Steph. = Lepidolejeunea integristipula

Hygrolejeunea graeffeana Steph. = Lejeunea lumbricoides

Hygrolejeunea procumbens (Mitt.) Steph. $\equiv$ Lejeunea procumbens

Jamesoniella contracta (Reinw., Blume et Nees) N.Kitag. 三 Cuspidatula contracta

Jamesoniella ovifolia (Schiffn.) Schiffn. = Denotarisia linguifolia

Jungermannia ariadne Taylor ex Lehm. $\equiv$ Solenostoma ariadne

Jungermannia flexicaulis Nees $\equiv$ Jamesoniella flexicaulis

Jungermannia haskarliana (Nees) Mitt. 三Solenostoma haskarlianum

Jungermannia sciurea Nees $\equiv$ Schistochila sciurea 
Jungermannia tetradactyla Hook.f. et Taylor $\equiv$ Telaranea tetradactyla

Jungermannia tetragona Lindenb. $\equiv$ Solenostoma tetragonum

Jungermannia truncata Nees $\equiv$ Solenostoma truncatum

Kurzia bisetula (Steph.) Grolle $\equiv$ Telaranea bisetula

Lejeunea apiculata Schiffn. nom. inval. = Colura leratii

Lejeunea caespitosa Lindenb. = Lejeunea capensis

Lejeunea convexifolia Mitt. $\equiv$ Pycnolejeunea convexifolia

Lejeunea crassitexta (J.B.Jack et Steph.) R.M.Schust. $\equiv$ Metalejeunea crassitexta

Lejeunea cucullata (Reinw., Blume et Nees) Nees nom. illeg. $\equiv$ Metalejeunea cucullata

Lejeunea cucullata (Reinw., Blume et Nees) Nees $\equiv$ Metalejeunea cucullata

Lejeunea eulopha Taylor $\equiv$ Lopholejeunea eulopha

Lejeunea graeffei J.B.Jack et Steph. ex P.Geissler et Bischl. nom. inval. = Lepidolejeunea graeffei

Lejeunea hawaiiensis Gottsche ex Mitt. = Lepidolejeunea involuta

Lejeunea integristipula (Mizut.) J.B.Jack et Steph. ex P.Geissler et Bischl. nom. inval. = Lepidolejeunea integristipula

Lejeunea meyeniana Nees, Lindenb. et Gottsche $\equiv$ Cheilolejeunea meyeniana

Lejeunea polyantha Mitt. nom. illeg. = Cololejeunea polyantha

Lejeunea polyrhiza auct. nom. illeg. = Leptolejeunea epiphylla

Lejeunea superba Mont. $\equiv$ Colura superba

Lejeunea superba var. apiculata Schiffn. nom. inval. = Colura leratii

Lejeunea tripuncta Mitt. $\equiv$ Leptolejeunea tripuncta

Lejeunea uvifera Mont. = Lejeunea flava

Lepicolea loriana Steph. $=$ Lepicolea rara

Lepidozia bisetula Steph. $\equiv$ Telaranea bisetula

Lepidozia lindenbergii Gottsche $\equiv$ Telaranea lindenbergii

Lepidozia pruinosa Herzog $\equiv$ Telaranea pruinosa

Lepidozia tenera Steph. = Telaranea wallichiana

Leptocolea goebelii (Gottsche ex Schiffn.) A.Evans = Cololejeunea trichomanis

Leptocolea vitiensis (Mitt.) E.O.Campb. nom. illeg. = Cololejeunea polyantha

Leptolejeunea radiata (Mitt.) Steph. = Leptolejeunea maculata

Leptolejeunea rhombifolia Steph. = Leptolejeunea maculata

Leptolejeunea schiffneri (Steph. ex Schiffn.) Steph. = Leptolejeunea maculata

Leptolejeunea schiffneri (Steph.) Steph. nom. inval. = Leptolejeunea maculata

Leptolejeunea subacuta Steph. ex A.Evans = Leptolejeunea elliptica

Lophocolea graeffei J.B.Jack et Steph. $\equiv$ Heteroscyphus graeffei

Lophocolea muricata (Lehm.) Nees $\equiv$ Chiloscyphus muricatus

Lophocolea parva Steph. $\equiv$ Chiloscyphus parvus

Lophocolea rectangulata Mitt. $\equiv$ Chiloscyphus samoanus

Lopholejeunea contractilis (Mitt.) Steph. = Caudalejeunea recurvistipula

Lopholejeunea immersa (Mitt.) Steph. = Lopholejeunea eulopha

Lopholejeunea multiflora J.B.Jack et Steph. = Lopholejeunea nigricans 
Lopholejeunea tecta (Mitt.) Steph. = Lopholejeunea plicatiscypha

Madotheca stangeri Lindenb. et Gottsche = Porella elegantula

Madotheca viridissima Mitt. ex Steph. = Porella viridissima

Marchantia nitida Lehm. et Lindenb. = Marchantia paleacea subsp. paleacea

Marchantia samoana Steph. = Marchantia vitiensis

Marsupidium urvilleanum (Mont.) Mitt. = Tylimanthus saccatus

Mastigobryum australe (Mont.) Lindenb. = Bazzania tridens

Mastigobryum caudistipulum Steph. $\equiv$ Bazzania caudistipula

Mastigobryum combinatum J.B.Jack et Steph. $\equiv$ Bazzania combinata

Mastigobryum hamatifolium Steph. $\equiv$ Bazzania hamatifolia

Mastigobryum paradoxum Sande Lac. $\equiv$ Bazzania paradoxa

Mastigobryum uncigerum (Reinw., Blume et Nees) Nees $\equiv$ Bazzania uncigera

Mastigobryum vitiense Mitt. ex Steph. = Bazzania vitiana

Mastigolejeunea guahamensis (Lindenb.) Steph. = Mastigolejeunea auriculata

Mastigolejeunea humilis (Gottsche) Schiffn. = Mastigolejeunea auriculata

Mastigolejeunea taitica (Gottsche ex Steph.) Gottsche ex Steph. = Mastigolejeunea virens

Mastigophora angustisecta Herzog = Mastigophora diclados

Megaceros monospirus Steph. = Megaceros flagellaris

Metzgeria decipiens (C.Massal.) Schiffn. = Metzgeria ciliata

Metzgeria densiseta Steph. = Metzgeria furcata

Metzgeria hamata (Mitt.) Lindb. nom. illeg. $\equiv$ Metzgeria procera

Metzgeria minor (Schiffn.) Kuwah. = Metzgeria lindbergii

Metzgeria oceanica Kuwah. = Metzgeria lindbergii

Metzgeria papulosa Steph. = Metzgeria leptoneura

Metzgeria parvipapulosa Kuwah. = Metzgeria leptoneura

Metzgeria pectinata Steph. $=$ Metzgeria lindbergii

Metzgeriopsis pusilla K.I.Goebel = Cololejeunea metzgeriopsis

Microlejeunea albicans (Nees) Steph. = Metalejeunea cucullata

Microlejeunea crassitexta J.B.Jack et Steph. $\equiv$ Metalejeunea crassitexta

Microlejeunea cucullata (Reinw., Blume et Nees) J.B.Jack et Steph. $\equiv$ Metalejeunea cucullata

Microlejeunea samoana Steph. = Lejeunea patersonii

Moerckia radiculosa Schiffn. $\equiv$ Sandeothallus radiculosus

Neohattoria parhamii R.M.Schust. $\equiv$ Frullania parhamii

Noguchia opposita (Reinw., Blume et Nees) Inoue $\equiv$ Plagiochilion oppositum

Paraschistochila graeffeana (J.B.Jack et Steph.) R.M.Schust. = Schistochila aligera

Phaeoceros laevis subsp. carolinianus (Michx.) Prosk. $\equiv$ Phaeoceros carolinianus

Phragmicoma bilabiata Mitt. $\equiv$ Archilejeunea bilabiata

Phragmicoma calcarata (Mont.) Mitt. nom. illeg. = Mastigolejeunea calcarata

Phragmicoma contractilis Mitt. $=$ Caudalejeunea recurvistipula

Phragmicoma eulopha (Taylor) Mitt. 三Lopholejeunea eulopha

Phragmicoma plana (Sande Lac.) Mitt. = Thysananthus retusus 
Phragmicoma renistipula Mitt. $\equiv$ Lopholejeunea renistipula

Physiotium giganteum (F.Weber) Lindb. $\equiv$ Pleurozia gigantea

Physocolea polyantha (Mitt.) Steph. = Cololejeunea polyantha

Plagiochila alta Steph. = Plagiochila arbuscula

Plagiochila auriculata Mitt. = Plagiochila bantamensis

Plagiochila belangeriana Lindenb. = Plagiochila arbuscula

Plagiochila belangeriana var. heterospina (Steph. ex Herzog) E.O.Campb. = Plagiochila arbuscula

Plagiochila blepharophora $\equiv$ vitilevuana Schiffn. = Plagiochila vitiensis

Plagiochila brotheri Steph. $=$ Plagiochila vitiensis

Plagiochila combinata Mitt. $\equiv$ Plagiochilion combinatum

Plagiochila dendroides (Nees) Lindenb. $\equiv$ Chiastocaulon dendroides

Plagiochila geminifolia Mitt. = Plagiochilion oppositum

Plagiochila heterospina Steph. ex Herzog nom. illeg. = Plagiochila arbuscula

Plagiochila longispica Mitt. = Plagiochila chauviniana

Plagiochila opposita (Reinw., Blume et Nees) Lindenb. $\equiv$ Plagiochilion oppositum

Plagiochila ovalava Steph. $=$ P. teysmannii

Plagiochila parvisacculata Steph. = Plagiochila bantamensis

Plagiochila powellii Mitt. ex Steph. = Plagiochila javanica

Plagiochila seemannii Mitt. = Plagiochila sandei

Plagiochila seemannii var. perspinosa Herzog = Plagiochila sandei

Plagiochila tahitensis Ångstr. = Plagiochila vitiensis

Plectocolea ariadne (Taylor ex Lehm.) Mitt. 三 Solenostoma ariadne

Plectocolea haskarliana (Nees) Mitt. $\equiv$ Solenostoma haskarlianum

Plectocolea tetragona (Lindenb.) Amakawa $\equiv$ Solenostoma tetragonum

Porella hebridensis (Steph.) E.O.Campb. = Porella viridissima

Porella stangeri (Lindenb. et Gottsche) Trevis. = Porella elegantula

Ptilidium sciureum (Nees) Nees $\equiv$ Schistochila sciurea

Ptychocoleus pycnocladus (Taylor) Steph. 三Acrolejeunea pycnoclada

Pycnolejeunea bidentula (Steph.) Steph. nom. inval. 三 Lepidolejeunea bidentula

Pycnolejeunea bidentula Steph. $\equiv$ Lepidolejeunea bidentula

Pycnolejeunea ceylanica (Gottsche) Schiffn. 三 Cheilolejeunea ceylanica

Pycnolejeunea convexistipa (Mitt.) Steph. nom. illeg. P Pycnolejeunea convexifolia

Pycnolejeunea decurvifolia (Steph.) Steph. = Lepidolejeunea bidentula

Pycnolejeunea falsinervis (Sande Lac.) Schiffn. $\equiv$ Cheilolejeunea falsinervis

Pycnolejeunea graeffei (J.B.Jack et Steph.) Horik. 三 Lepidolejeunea graeffei

Pycnolejeunea graeffei (J.B.Jack et Steph.) Verd. nom. inval. $\equiv$ Lepidolejeunea graeffei

Pycnolejeunea integristipula J.B.Jack et Steph. $\equiv$ Lepidolejeunea integristipula

Pycnolejeunea meyeniana (Nees, Lindenb. et Gottsche) Steph. $\equiv$ Cheilolejeunea meyeniana

Pycnolejeunea setifera Steph. = Cheilolejeunea gardneri

Pycnolejeunea trapezia (Nees) Schiffn. $\equiv$ Cheilolejeunea trapezia 
Radula miqueliana Taylor $=$ Radula retroflexa

Radula spicata Mitt. = Radula formosa

Riccardia fasciculata (Steph.) Steph. nom. illeg. $\equiv$ Riccardia pectinata var. fasciculata

Riccardia lobata Schiffn. = Lobatiriccardia coronopus

Riccardia maxima Schiffn. $\equiv$ Aneura maxima

Riccardia samoana (Steph.) H.A.Mill. = Riccardia alba

Riccardia serrulata Schiffn. = Riccardia heteroclada

Riccardia vitiensis (Steph.) E.O.Campb. = Riccardia alba

Ricciella linearis Schiffn. $\equiv$ Riccia linearis

Saccogynidium jugatum (Mitt.) Grolle = Saccogynidium rigidulum

Sarcomitrium plumosum Mitt. $\equiv$ Riccardia plumosa

Schiffneriolejeunea haskarliana (Gottsche) Gradst. 三 Schiffneriolejeunea tumida var. haskarliana

Schistochila flavicans Steph. $=$ Schistochila caledonica

Schistochila graeffeana J.B.Jack et Steph. = Schistochila aligera

Schistochila heterodonta (Colenso) Steph. $=$ Schistochila repleta

Schistochila philippinensis (Mont.) Steph. $=$ Schistochila aligera

Schistochilaster graeffeanus (J.B.Jack et Steph.) H.A.Mill. = Schistochila aligera

Schistochilaster philippinensis (Mont.) H.A.Mill. = Schistochila aligera

Schusterella parhamii (R.M.Schust.) S.Hatt., Sharp et Mizut. $\equiv$ Frullania parhamii

Solenostoma hasskarlianum (Nees) R.M.Schust. nom. inval. $\equiv$ Solenostoma haskarlianum

Spruceanthus marianus (Gottsche) Mizut. 三 Cheilolejeunea mariana

Symbiezidium bacciferum (Taylor) Steph. = Symbiezidium transversale var. transversale

Symbiezidium integristipulum (J.B.Jack et Steph.) Steph. 三Lepidolejeunea integristipula

Symphyogyna vitiensis J.B.Jack et Steph. = Symphyogynopsis gottscheana

Symphyogynopsis filicum (Nadeaud) Grolle = Symphyogynopsis gottscheana

Taxilejeunea cuculliflora Steph. 三Lejeunea cuculliflora

Taxilejeunea gracilipes (Taylor) Steph. ELejeunea gracilipes

Taxilejeunea lumbricoides (Nees) Schiffn. $\equiv$ Lejeunea lumbricoides

Taxilejeunea umbilicata (Nees) J.B.Jack et Steph. $\equiv$ Lejeunea umbilicata

Thysananthus fruticosus (Lindenb. et Gottsche) Schiffn. 三 Dendrolejeunea fruticosa

Thysananthus planus Sande Lac. $=$ Thysananthus retusus

Thysananthus sinclairii (Mitt.) Steph. $\equiv$ Lejeunea sinclairii

Thysanolejeunea fruticosa (Lindenb. et Gottsche) Steph. $\equiv$ Dendrolejeunea fruticosa

Thysanolejeunea spathulistipa (Reinw., Blume et Nees) Steph. $\equiv$ Thysananthus spathulistipus

Treubia bracteata Steph. = Treubia lacunosa

Treubia bracteata Steph. $\equiv$ Treubia insignis subsp. bracteata

Tylimanthus urvilleanus (Mont.) Hässel et Solari = Tylimanthus saccatus 


\section{Acknowledgments}

The financial assistance of the National Geographic Committee for Research and Exploration (Grant No. 8247-07) and Global Biological Information Facility (GBIF) Seed Money Award No. 2007/41 is gratefully acknowledged. We wish to also thank the Biodiversity Synthesis Center (BioSynC) at The Field Museum - a component of the Encyclopedia of Life $(E O L)$ - which sponsored an international liverwort meeting and workshop that contributed to this checklist. The authors gratefully acknowledge the valuable assistance, guidance, and logistical support provided by the Suva Herbarium, and especially Marika Tuiwawa and Alifereti Naikatini.

\section{References}

Arnell S (1958) Hepatics from Tristan da Cunha. Results of the Norwegian Scientific Expedition to Tristan da Cunha, 1937-1938 42: 1-76.

Austin CF (1872) New Hepaticae. Bulletin of the Torrey Botanical Club 3: 9-18.

Bai X-L (2002) Frullaniaceae flora of Yunnan, China. Chenia 7: 1-27.

Bapna KR \& Kachroo P (2000) Hepaticology of India I. (Himanshu Publications: Udaipur).

Bapna KR \& Kachroo P (2000b) Hepaticology of India II. (Himanshu Publications: Udaipur).

Bischler H, Miller HA \& Bonner CEB (1961) [1962] Studies in Lejeuneaceae III. A historical account of Lejeunea cucullata (Reinwardt, Blume \& Nees) Nees and its varieties. Nova Hedwigia 3: 445-462.

Bischler-Causse H (1989) Marchantia L. The Asiatic and Oceanic taxa. Bryophytorum Bibliotheca 38: $1-317$.

Bizot M \& Pócs T (1974) East African Bryophytes I. Egri tanárképzö föiskola tudományos közleményei. Acta Academiae Paedagogicae Agriensis 12: 383-449.

Bonner CEB (1962a) Index Hepaticarum. Pars I. Plagiochila (Dum.) Dum. (J. Cramer: Weinheim).

Bonner CEB (1962b) Index Hepaticarum. Pars II. Achiton to Balantiopsis. (J. Cramer: Weinheim).

Bonner CEB (1963a) Index Hepaticarum. Pars III. Barbilophozia to Ceranthus. (J. Cramer: Weinheim).

Bonner CEB (1963b) Index Hepaticarum. Pars IV. Ceratolejeunea to Cystolejeunea. (J. Cramer: Weinheim).

Bonner CEB (1965) Index Hepaticarum. Pars V. Delavayella to Geothallus. (J. Cramer: Weinheim).

Bonner CEB (1966) Index Hepaticarum. Pars VI. Goebeliella to Jubula. (J. Cramer: Weinheim).

Bonner CEB (1977) Index Hepaticarum. Pars VIIa. Supplementum A-C. (J. Cramer: Vaduz).

Brown EA \& Braggins JE (1989) A revision of the genus Riccardia S. F. Gray in New Zealand with notes on the genus Aneura Dum. Journal of the Hattori Botanical Laboratory 66: 1-132.

Campbell EO (1971) Liverworts collected in Fiji by A. C. Smith and W. Greenwood. Journal of the Royal Society of New Zealand 1: 7-30.

Castle H (1950) A revision of the genus Radula. Part II. Subgenus Acroradula. Section 2. Amentulosae. The Bryologist 53: 253-275.

Castle H (1965) A revision of the genus Radula. Part II. Subgenus Acroradula. Section 9. Densifoliae. Revue Bryologique et Lichénologique 33: 328-398.

Castle H (1966) A revision of the genus Radula. Part II. Subgenus Acroradula. Section 10. Ampliatae. Revue Bryologique et Lichénologique 34: 1-81.

Chantanaorrapint S, Boonkered T \& Thaithong O (2004) Checklist of bryophytes at the summit of Khao Luang, Huai Yang Waterfall National Park, Prachuap Khiri Khan Province, Thailand. Natural history bulletin of the Siam society 52: 163-179.

Clark L \& Frye TC (1948) Frullania intermedia. The Bryologist 51: 52-54.

Costa DP (2008) Metzgeriaceae. Flora Neotropica 102: 1-169.

Crosby MR \& Engel JJ (2006) Index of Hepatics. (Hattori Botanical Laboratory: Nichinan). 
Damsholt K (2002) Illustrated flora of Nordic liverworts and hornworts. (Nordic Bryological Society: Lund).

DoE (Department of the Environment) (1997) Convention on Biological Diversity 1997. National Report to the Conference of the Parties by the Republic of Fiji. (Department of the Environment, Ministry of Local Government, Housing and Environment: Suva, Fiji).

Eggers J (2006) New bryophyte taxon records for tropical countries 6. Tropical Bryology 27: $107-111$.

Eggers J \& Pócs T (2010) Epiphylle Lebermoose aus Samoa. Auf den Spuren von Eduard Graeffe in der Farn-Abteilung des Herbarium Hamburgense. Berichte des Botanischen Vereins zu Hamburg 25: 3-11.

Eifrig H (1936) [1937] Monographische Studien über die indomalayischen Arten von Taxilejeunea. Annales Bryologici 9: 73-114.

Engel JJ (1976) Metzgeria hamata Lindb., an illegitimate name of Hepaticae. Lindbergia 3: 219-220.

Engel JJ (1992) Index Hepaticarum-Supplementum - 1984-1985. Taxon 41: 253-306.

Engel JJ \& Schuster RM (1985) [1984] An overview and evaluation of the genera of Geocalycaceae subfamily Lophocoleoideae (Hepaticae). Nova Hedwigia 39: 385-463.

Engel JJ \& Smith Merrill GL (1996) Studies of New Zealand Hepaticae. 14-19. Kurzia and Lepicolea. Journal of the Hattori Botanical Laboratory 80: 217-231.

Engel JJ \& Smith Merrill GL (2004) Austral Hepaticae 35. A taxonomic and phylogenetic study of Telaranea (Lepidoziaceae), with a monograph of the genus in temperate Australasia and commentary on extra-Australasian taxa. Fieldiana, Botany, n.s. 44: 1-265.

Evans AW (1917) The American species of Marchantia. Transactions of the Connecticut Academy of Arts and Sciences 21: 253-261.

Evans AW (1925) A taxonomic study of Hymenophytum. Bulletin of the Torrey Botanical Club 52: 491-506.

Evans AW (1932) Some representative species of Bazzania from Sumatra. Papers of the Michigan academy of Science, Arts and Letters 17: 69-118.

Feldberg K, Váňa J, Hentschel J \& Heinrichs J (2010) Currently accepted species and new combinations in Jamesonielloideae (Adelanthaceae, Jungermanniales). Cryptogamie, Bryologie 31: 141-146.

Frye TC \& Clark L (1944) [1943] Hepaticae of North America part II. University of Washington Publications in Biology 6: 163-336.

Fulford M (1963) Manual of the leafy Hepaticae of Latin America I. Memoirs of the New York Botanical Garden 11: 1-172.

Furuki T (1991) A taxonomical revision of the Aneuraceae (Hepaticae) of Japan. Journal of the Hattori Botanical Laboratory 70: 293-397.

Furuki T (2002) Studies on the bryophyte flora of Vanuatu. 5. Metzgeriales and Marchantiales (Hepaticae). Annals of the Tsukuba Botanical Garden 21: 95-101.

Furuki T (2006) Taxonomical studies of the family Aneureaceae (Hepaticae) based on the Philippine collections made by Dr. and Mrs. A. J. Sharp and Dr. Z. Iwatsuki. Journal of the Hattori Botanical Laboratory 100: 89-99.

Gao C \& Bai X-L (2001) A synoptic revision of family Jungermanniaceae (Hepaticae) in China including some taxa nova. Philippine Scientist 38: 111-170.

Gao C, Cao T \& Lai MJ (2001) The genus Saccogynidium (Geocalycaceae, Hepaticae) in China. The Bryologist 104: 126-129.

Geissler P \& Bischler H (1985) Index Hepaticarum vol. 10. Lembidium to Mytilopsis. (J. Cramer: Berlin).

Geissler P \& Bischler H (1987) Index Hepaticarum vol. 8/9. Jungermannia to Lejeunites. (J. Cramer: Berlin).

Geissler P \& Bischler H (1989) Index Hepaticarum vol. 11. Naiadea to Pycnoscenus. (J. Cramer: Berlin). 
Geissler P \& Bischler H (1990) Index Hepaticarum vol. 12. Racemigemma to Zoopsis. (J. Cramer: Berlin).

Geissler P \& Gradstein SR (1994) On the identity of Phragmicoma lehmanniana Nees and other species described in the genus Phramicoma (Studies in Lejeuneaceae subfam. Pthycanthoideae 23). Journal of the Hattori Botanical Laboratory 75: 201-209.

Gibbs LS (1909) A contribution to the montane flora of Fiji (including Cryptogams), with ecological notes. Journal of the Linnean Society, Botany 39: 130-212.

Gradstein SR (1975) A taxonomic monograph of the genus Acrolejeunea (Hepaticae). Bryophytorum Bibliotheca 4: 1-162.

Gradstein SR \& Costa DP (2003) The hepaticae and Anthocerotae of Brazil. Memoirs of the New York Botanical Garden 87: 1-316.

Gradstein SR, He X-L, Piippo S \& Mizutani M (2002) Bryophyte flora of the Huon Peninsula, Papua New Guinea. LXVIII. Lejeuneaceae subfamily Ptychanthoideae (Hepaticae). Acta Botanica Fennica 174: 1-88.

Gradstein SR \& van Beek J (1985) A revision of the genus Symbiezidium Trevis. Nova Hedwigia, Beiheft 80: 221-249.

Gradstein SR, Wilson R, Ilkiu-Borges AL \& Heinrichs J (2006) Phylogenetic relationships and neotenic evolution of Metzgeriopsis (Lejeuneaceae) based on chloroplast DNA sequences and morphology. Journal of the Linnean Society. Botany 151: 293-308.

Grolle R (1965) Lebermoose aus Neuguinea. I. Journal of the Hattori Botanical Laboratory 28: $43-54$.

Grolle R (1966) Lebermoose aus Neuguinea. 2. Zweite Fundliste. Journal of the Hattori Botanical Laboratory 29: 70-74.

Grolle R (1968) Einige ostmalesische Lebermoose. Nova Hedwigia 16: 147-159.

Grolle R (1969) Großdisjunktionen in artarealen Lateinamerikanischer Lebermoose. Monographiae biologicae 19: 562-582.

Grolle R (1971) Jamesoniella und Verwandte. Feddes Repertorium 82: 1-100.

Grolle R (1979) Miscellania Hepaticologica 181-190. Journal of the Hattori Botanical Laboratory 45: $173-183$.

Grolle R (1980) Zur kenntnis der Lebermoose von Samoa I. Wissenschaftliche Zeitschrift der Friedrich-Schiller-Universität Jena/Thüringen. Matematisch-naturwissenschaftliche Reihe 29: 637-648.

Grolle R (1982) Übersicht der Lejeuneaceae in Tasmanien. Wissenschaftliche Zeitschrift der Friedrich-Schiller-Universität Jena/Thüringen. Matematisch-naturwissenschaftliche Reihe 31: 207-227.

Grolle R (1988) Miscellanea Hepaticologica 261-270. Journal of the Hattori Botanical Laboratory 65: 403-410.

Grolle R (2002) Two new synonyms of Metzgeria furcata (L.) Dumort. Cryptogamie, Bryologie 23: $209-210$.

Grolle R \& Piippo S (1984) Bryophyte flora of the Huon Peninsula, Papua New Guinea. V. Lepidoziaceae subfam. Zoopsoideae and Cephaloziaceae subfam. Schiffnerioideae (Hepaticae). Annales Botanci Fennici 21: 299-307.

Grolle R \& Piippo S (1986) Bryophyte flora of the Huon Peninsula, Papua New Guinea. XVI. Pallaviciniaceae (Hepaticae). Acta Botanica Fennica 133: 59-79.

Grolle R \& Reiner-Drehwald ME (1999) Review of the genus Harpalejeunea (Lejeuneaceae) including the description of $H$. grandis, a new species from the páramos of Colombia. Journal of Bryology 21: 31-45.

Grolle R \& Schultze-Motel W (1972)[1973] Vorläufiges Verzeichnis der Lebermoose von Samoa. Journal of the Hattori Botanical Laboratory 36: 75-89.

Grolle R \& So ML (1999) Notes on Plagiochila subgenus Paraplagiochila (Hepaticae). Journal of Bryology 21: 197-199.

Guo W \& Zhang M-X (1999) The taxonomic study of Anthocerotaceae in Shaanxi, Northwest China. Chenia 6: 21-33. 
Haerida I, Gradstein SR \& Tjitrosoedirdjo SS (2010) Lejeuneaceae subfamily Ptychanthoideae (Hepaticae) in West Java. Gardens' Bulletin. Singapore 62: 59-111.

Hasegawa J (1984) Taxonomical studies on Asian Anthocerotae IV. A revision of the genera Anthoceros, Phaeoceros and Folioceros in Japan. Journal of the Hattori Botanical Laboratory 57: 241-272.

Hasegawa J (1986a) The Anthocerotae collected by Drs. Z. Iwatsuki and N. Kitagawa in New Caledonia and Fiji. Journal of the Hattori Botanical Laboratory 60: 379-390.

Hasegawa J (1986b) A collection of of the Anthocerotae from Seram and Ambon. Acta Phytotaxonomica et Geobotanica. [Shokubutsu bunrui chiri] 37: 9-15.

Hasegawa J (1993) Taxonomic studies on Asian Anthocerotae V. A short revision of Taiwanese Anthocerotae. Acta Phytotaxonomica et Geobotanica. [Shokubutsu bunrui chiri] 44: 97-112.

Hasegawa J (2000) Hattorioceros striatisporus (Hasegawa) Hasegawa newly found in Fiji. Bryological Research [Sentairui Kenkyu] 7: 273-275.

Hasegawa J (2002) Studies on the bryophyte flora of Vanuatu. 6. Anthocerotae. Annals of the Tsukuba Botanical Garden 21: 103-107.

Hässel de Menéndez GG \& Solari SS (1975) Bryophyta Hepaticopsida: Calobryales, Jungermanniales: Vetaformaceae, Balantiopsidaceae. Pp. 7-181 in Guerrera, S.A., Gamundi de Amos, I. \& Rabininovich de Halperin, D. (eds.), Flora Criptogámica de Tierra del Fuego. 15. (Funcación para la Educación, la Ciencia y la Cultura: Buenos Aires).

Hattori S (1966) Anthocerotae and Hepaticae. Pp. 501-536 in Hara H (ed.), The flora of eastern Himalaya. Results of the botanical expedition to eastern Himalaya organized by the University of Tokyo 1960 and 1963. (University of Tokyo Press: Tokyo).

Hattori S (1968) Hepaticae collected by F. Schmid in Ceylon and Pakistan. Candollea 23: 287-294.

Hattori S (1973) Notes on Asiatic species of the genus Frullania, Hepaticae, II. Journal of the Hattori Botanical Laboratory 37: 55-84.

Hattori S (1975a) Studies of the Asiatic species of the genus Porella (Hepaticae). V. Journal of the Hattori Botanical Laboratory 39: 269-276.

Hattori S (1975b) Notes on Asiatic species of the genus Frullania, Hepaticae. VII. Journal of the Hattori Botanical Laboratory 39: 277-313.

Hattori S (1975c) Bryophyta, Anthocerotae \& Hepaticae (in Ohashi H (ed), Flora of E Himalaya, Third report). Bulletin, University Museum, University of Tokyo. (Tokyo Daigaku. Sogo Kenkyu Shiryokan) 8: 206-242.

Hattori S (1975d) A supplementary note on Porella viridissima. Miscellanea Bryologica et Lichenologica 7: 46.

Hattori S (1977) Dr. H. Hürlimann's collections of New Caledonian Frullaniaceae. Journal of the Hattori Botanical Laboratory 43: 409-438.

Hattori S (1980) A revision of the subgenus Homotropantha of the genus Frullania, Hepaticae. Journal of the Hattori Botanical Laboratory 47: 165-236.

Hattori S (1981) Notes on the Pacific species of Frullanianiaceae (Hepaticae), I. Journal of the Hattori Botanical Laboratory 49: 359-383.

Hattori S (1982) A synopsis of New Guinean Frullania, Hepaticae. Journal of the Hattori Botanical Laboratory 51: 203-271.

Hattori S (1984) New Caledonian Frullaniaceae. Journal of the Hattori Botanical Laboratory 57: 405-426.

Hattori S (1985) A small collection of Frullania and Porella (Hepaticae) made on Viti Levu, Fiji Islands. Bulletin of the National Science Museum, Tokyo. Series B, Botany 11: 11-17.

Hattori S (1986a) A synopsis of New Caledonian Frullaniaceae. Journal of the Hattori Botanical Laboratory 60: 203-237.

Hattori S (1986b) Frullania collection made by Mr. H. Akiyama on Seram Island. Journal of the Hattori Botanical Laboratory 60: 239-253.

Hattori S (1986c) Porellaceae from New Caledonia. Journal of Japanese Botany [Shokubutsu kenkyu zasshi] 61: 185-192. 
Hattori S (1988) Frullania flora of Mt. Albert Edward, Papua New Guinea. Journal of the Hattori Botanical Laboratory 65: 411-453.

Hattori S \& Lin P-J (1985) A preliminary study of Chinese Frullania flora. Journal of the Hattori Botanical Laboratory 59: 123-169.

Hattori S \& Piippo S (1986) Bryophyte flora of the Huon Peninsula, Papua New Guinea. XV. Frullania (Frullaniaceae, Hepaticae). Acta Botanica Fennica 133: 25-58.

Hattori S, Sharp AJ \& Mizutani M (1972) Schusterella, a new genus of Jubulaceae (Hepaticae). Journal of Japanese Botany [Shokubutsu kenkyu zasshi] 20: 329-338.

Hattori S \& Streimann H (1985) A collection of Frullania taxa from Papua New Guinea. Journal of the Hattori Botanical Laboratory 59: 101-121.

He X-L (1999) A taxonomic monograph of the genus Pycnolejeunea (Lejeuneaceae, Hepaticae). Acta Botanica Fennica 163: 1-77.

Hentschel J, von Konrat MJ, Pócs T, Schäfer-Verwimp A, Shaw AJ, Schneider H \& Heinrichs J (2009) Molecular insights into the phylogeny and subgeneric classification of Frullania Raddi (Frullaniaceae, Porellales). Molecular Phylogenetics and Evolution 52: 142-156.

Herzog T (1938) Beiträge zur Kenntnis der Gattung Plagiochila II. Palaeotropische Arten. Hedwigia 78: 222-244.

Herzog T (1939) Studien über Drepanolejeunea IV. Annales Bryologici 12: 98-122.

Herzog T (1951a)[1952] Miscellanea bryologica. III. Memoranda societatis pro fauna et flora fennica 27: 92-110.

Herzog T (1950) [1951b] Miscellanea bryologica. II. Paleotropica. Memoranda societatis pro fauna et flora fennica 26: 37-66.

Herzog T (1953) Lebermoosen aus Neukaledonien gesammelt von Dr. O. H. Selling. Arkiv för Botanik, n.s. 2: 43-61.

Horikawa Y (1943) Notulae Hepaticologicae. Acta Phytotaxonomica et Geobotanica. [Shokubutsu bunrui chiri] 13: 212-214.

Hürlimann H (1960) Hepaticae aus dem Gebiete des südlichen Pazifik. I. Bauhinia 1: 251-260.

Hürlimann H (1968) Hepaticae aus dem Gebiete des südlichen Pazifik. II. Bauhinia 4: 73-84.

Hürlimann H (1974) Hepaticae aus dem Gebiete des südlichen Pazifik. III. Bauhinia 5: 59-68.

Hürlimann H (1976) Hepaticae aus dem Gebiete des südlichen Pazifik. IV. Bauhinia 5: 191-213.

Hürlimann H (1978) Hepaticae aus dem Gebiete des südlichen Pazifik. VI. Bauhinia 6: 293-305.

Hürlimann H (1983) Hepaticae aus dem Gebiete des südlichen Pazifik. VII. Bauhinia 7: 259-268.

Hürlimann H (1985) Hepaticae aus dem Gebiete des südlichen Pazifik. VIII. Bauhinia 8: 101118.

Hürlimann H (1987) Hepaticae aus dem Gebiete des südlichen Pazifik. IX. Bauhinia 8: 221-234.

Hürlimann H (1989) Hepaticae aus dem Gebiete des südlichen Pazifik. X. Bauhinia 9: 153-170.

Hürlimann H (1991) Hepaticae aus dem Gebiete des südlichen Pazifik. XI. Bauhinia 9: 257-264.

Hürlimann H (1993) Hepaticae aus dem Gebiete des südlichen Pazifik. XII. Bauhinia 11:3-17.

Hürlimann H (1995) Hepaticae aus dem Gebiete des südlichen Pazifik. XIII. Bauhinia 11: $159-175$.

Hürlimann H (1998) Hepaticae aus dem Gebiete des südlichen Pazifik XIV. Bauhinia 12: $109-119$.

Inoue H (1958) The family Plagiochilaceae of Japan and Formosa. II. Journal of the Hattori Botanical Laboratory 20: 54-106.

Inoue H (1970) Plagiochilaceae notes, I. On some species of Plagiochila from south Pacific Islands. Journal of the Hattori Botanical Laboratory 33: 305-316.

Inoue H (1972c) Distribution and speciation of certain sections of Plagiochila. Journal of the Hattori Botanical Laboratory 35: 25-30.

Inoue H (1981) Notes on the Plagiochilaceae, XI. A review of the species of Plagiochila (Dum.) Dum. from Samoa. Journal of the Hattori Botanical Laboratory 49: 335-357.

Inoue H (1982) Studies on Taiwan Hepaticae. IV. Plagiochilaceae. Bulletin of the National Science Museum, Tokyo. Series B, Botany 8: 125-144.

Inoue H (1984) The genus Plagiochila in Southeast Asia. (Academic Scientific Books: Tokyo). 
Inoue H (1986) Notes on the Plagiochilaceae, XIII. The genus Plagiochila (Dum.) Dum. in mainland Australia. Journal of the Hattori Botanical Laboratory 60: 357-378.

Inoue H \& Iwatsuki Z (1969) Bryophytes of the Bonin Islands and the Volcano Islands (1). Bulletin of the National Science Museum, n.s. 12: 291-309.

Inoue H \& Miller HA (1965) Hepaticae from Kusaie, Caroline Islands. Bulletin of the National Science Museum, Tokyo. Series B, Botany 8: 139-160.

Jack JB \& Stephani F (1894) Hepaticae in insulis Vitibuensibus et Samoanis a Dre Ed. Graeffe anno 1864 lectae. Botanisches Centralblatt 60: 97-109.

Jones EW (1972) African hepatics XXIII. Some species of Lejeunea. Journal of Bryology 7: 23-45. Jovet-Ast S (1951) Hépatiques des Nouvelles-Hebrides. Recoltes de E. Aubert de la Rüe, 1934. Revue Bryologique et Lichénologique 20: 96-98.

Jovet-Ast S (1954) Le genre Colura. Hepatiques, Lejeuneaceae, Diplasiae (supplement). Revue Bryologique et Lichénologique 23: 1-22.

Jovet-Ast S \& Tixier P (1962) Hépatiques du Vietnam. II. Revue Bryologique et Lichénologique 31: 23-33.

Kitagawa N (1967) Studies on the Hepaticae of Thailand. I. The genus Bazzania, with general introduction. Journal of the Hattori Botanical Laboratory 30: 249-270.

Kitagawa N (1972) [1973a] Miscellaneous notes on little-known species of Hepaticae, 1-25. Journal of the Hattori Botanical Laboratory 36: 444-454.

Kitagawa N (1973b) Miscellaneous notes on little-known species of Hepaticae, 26-50. Journal of the Hattori Botanical Laboratory 37: 263-273.

Kitagawa N (1978) The Hepaticae of Thailand collected by Dr. A. Touw (I). Acta Phytotaxonomica et Geobotanica. [Shokubutsu bunrui chiri] 29: 47-64.

Kitagawa N (1979) The Hepaticae of Thailand collected by Dr. A. Touw (II). Acta Phytotaxonomica et Geobotanica. [Shokubutsu bunrui chiri] 30: 31-40.

Kitagawa N (1981) Miscellaneous notes on little-known species of Hepaticae, 51-70. Hikobia, supplement 1: 67-72.

Koike N (1994) Frullania taxa of some islands of Micronesia. Journal of the Hattori Botanical Laboratory 75: 183-192.

Koponen T, Enroth J, Fang J-M, Huttunen S, Hyvönen J, Ignatov M, Juslén A, Lai M-J, Piippo S, Potemkin A \& Rao P (2000) Bryophyte flora of Hunan Province, China. 1. Bryophytes from Mangshan Nature Reserve and Wulingyuan Global Cultural Heritage Area. Annales Botanci Fennici 37: 11-40.

Kuwahara Y (1960) [1961] The genus Metzgeria in Pacific Oceania. Journal of the Hattori Botanical Laboratory 23: 3-28.

Kuwahara Y (1965) The Metzgeriaceae of Mt. Kinabalu, North Borneo. Journal of the Hattori Botanical Laboratory 28: 166-170.

Kuwahara Y (1966) The family Metzgeriaceae in north and south east Asia, Pacific Ocean, Australia and New Zealand. Revue Bryologique et Lichénologique 34: 191-239.

Kuwahara Y (1968) Studies of a recent collection of Metzgeriaceae in the Philippines. Journal of the Hattori Botanical Laboratory 31: 159-175.

Kuwahara Y (1969) Taxonomic accounts of "the hair-falcate" species of the hepatic genus Metzgeria. Nova Hedwigia 17: 359-368.

Kuwahara Y (1982) Studies on Colombian Cryptogams XV. On high Andean Metzgeria collected by Dr. Antoine M. Cleef in 1972 and 1973. Proceedings, Koninklijke Nederlandse Akademie van Wetenschappen. Series C, biological and medical sciences 85(3): 357-380.

Kuwahara Y (1984) Synopsis of section Biseria subg. Metzgeria Kuw. genus Metzgeria Raddi (Hepaticae). Hikobia 9: 31-42.

Kuwahara Y (1984) [1985] Annotated key to the species of the Metzgeriaceae from Japan, East Himalayas, Tropical East Asia, and Tropical Oceania. Nova Hedwigia 40: 273-285.

Lee GE, Damanhuri A, Latiff A \& Gradstein SR (2011) A taxonomic treatment of Lejeunea discreta, L. eifrigii and L. sordida, new to peninsular Malaysia. Acta Biologica Plantarum Agriensis 1: 37-51. 
Long DG \& Grolle R (1990) Hepaticae of Bhutan II. Journal of the Hattori Botanical Laboratory 68: 381-440.

McNeill J, Barrie FR, Burdet HM, Demoulin V, Hawksworth DL, Marhold K, Nicolson DH, Prado J, Silva PC, Skog JE, Wiersema JH \& Turland NJ (2006) International Code of Botanical Nomenclature (Vienna Code) adopted by the Seventeenth International Botanical Congress Vienna, Austria, July 2005. Regnum Vegetabile 146: 1-260.

Meagher D (2010) Studies on Bazzania 2. Seven poorly known species from Australia. Nova Hedwigia 90: 395-411.

Miller HA (1960) A preliminary list of Micronesian Bryophytes. The Bryologist 63: 116-125.

Miller HA (1968) Herberta notes. Journal of the Hattori Botanical Laboratory 31: 247-250.

Miller HA, Bonner CEB \& Bischler H (1962) [1963b] Studies in Lejeuneaceae V. Microlejeunea in Pacific Oceania. Nova Hedwigia 4: 551-561.

Miller HA, Whittier HO \& Bonner CEB (1963a) Bryoflora of the atolls of Micronesia. Nova Hedwigia, Beiheft 11: 1-89.

Miller HA, Whittier HO \& Whittier BA (1983) Prodromus Florae Hepaticarum Polynesiae. Bryophytorum Bibliotheca 25: 1-423.

Mitten W (1861) Musci et hepaticae Vitiensis. Bonplandia 9: 365-367.

Mitten W (1862) Musci et Hepaticae Vitiensis. Bonplandia 10: 19.

Mitten W (1873) Jungermanniae and Marchantiae. Pp. 325-453 in Seeman B (ed.), Flora vitiensis, part 10. (Reeve: London).

Mittermeier RA, Gil PR, Hoffman M, Pilgrim J, Brooks T, Mittermeier CG, Lamoreux J \& da Fonseca GAB. (2005) Hotspots Revisited: Earth's Biologically Richest and Most Threatened Terrestrial Ecoregions (Cemex, Conservation International and Agrupacion Sierra Madre, Monterrey, Mexico

Mizutani M (1967) Studies of little known Asiatic species of Hepaticae in the Stephani Herbarium. 3. On some little known species of Cheilolejeunea, Euosmolejeunea and Pycnolejeunea. Journal of the Hattori Botanical Laboratory 30: 171-180.

Mizutani M (1972) Studies of little known Asiatic species of Hepaticae in the Stephani Herbarium. 7. Some little known species of the subfamily Lejeuneoideae of the Lejeuneaceae. Journal of the Hattori Botanical Laboratory 35: 399-411.

Mizutani M (1976) Studies of little known Asiatic species of Hepaticae in the Stephani Herbarium. 9. Some little known species of the family Lejeuneaceae. Journal of the Hattori Botanical Laboratory 40: 441-446.

Mizutani M (1977)[1978b] Lejeuneaceae from the Philippines. Journal of the Hattori Botanical Laboratory 43: 127-136.

Mizutani M (1978b) Lejeuneaceae from the Ishigaki and Iriomote Islands of Ryukyu Archipelago. Journal of the Hattori Botanical Laboratory 44: 121-136.

Mizutani M (1988) Notes on the Lejeuneaceae. 14. Asiatic species of the genus Caudalejeunea. Journal of the Hattori Botanical Laboratory 64: 389-399.

Montagne JFC (1843) Quatrieme centurie de plantes cellulaires exotiques nouvelles, décades I-VI. Annales des Sciences Naturelles, Botaniqe, ser. 2 19: 238-266.

Nair MC, Rajesh KP \& Madhusoodanan PV (2005) Little known Plagiochila (Dum.) Dum. (Plagiochilaceae: Hepaticae) from India. Geophytology 35: 39-44.

Pfeiffer T (2000) Molecular relationship of Hymenophyton species (Metzgeriideae, Hepaticophytina) in New Zealand and Tasmania. Studies in austral temperate rain forest bryophytes 5. New Zealand Journal of Botany 38: 415-424.

Piippo S (1984a) Bryophyte flora of the Huon Peninsula, Papua New Guinea. III. Haplomitriaceae, Lepicoleaceae, Herbertaceae, Pseudolepicoleaceae, Trichocoleaceae, Schistochilaceae, Balantiopsaceae, Pleuroziaceae and Porellaceae (Hepaticae). Annales Botanci Fennici 21: 21-48. 
Piippo S (1984b) Bryophyte flora of the Huon Peninsula, Papua New Guinea. VI. Lepidoziaceae subfam. Lepidozioideae, Calypogeiaceae, Adelanthaceae, Cephaloziaceae subfam. Cephalozioideae and subfam. Odontoschismatoideae and Jubulaceae (Hepaticae). Annales Botanci Fennici 21: 309-335.

Piippo S (1985) Bryophyte flora of the Huon Peninsula, Papua New Guinea. XII. Geocalycaceae (Hepaticae). Acta Botanica Fennica 131: 129-167.

Piippo S (1986) A monograph of Lepidolejeunea and Luteolejeunea (Lejeuneaceae: Hepaticae). Acta Botanica Fennica 132: 1-69.

Piippo S (1988) Bryophyte flora of the Huon Peninsula, Papua New Guinea. XXIII. Treubiaceae, Allisoniaceae and Makinoaceae (Metzgeriales, Hepaticae). Annales Botanici Fennici 25: 159_ 164.

Piippo S (1989a) Bryophyte flora of the Huon Peninsula, Papua New Guinea.XXX. Plagiochilaceae (Hepaticae). Annales Botanci Fennici 26: 183-236.

Piippo S (1989b) The bryophytes of Sabah (North Borneo) with specieal reference to hte BRYOTROP transect of Mount Kinabalu. III. Geocalycaceae (Hepaticae). Willdenowia 18: 513-527.

Piippo S (1991) Bryophyte flora of the Huon Peninsula, Papua New Guinea. XXXIX. Fossombronia (Fossombroniaceae) and Metzgeria (Metzgeriaceae). Annales Botanci Fennici 143: 1-22.

Piippo S (1992) Bryophyte flora of the Huon peninsula, Papua New Guinea. LI. Additions and corrections to the Geocalycaceae (Hepaticae). Annales Botanci Fennici 29: 243-248.

Piippo S (1993) Bryophyte flora of the Huon Peninsula, Papua New Guinea. LIV. Anthocerophyta. Acta Botanica Fennica 148: 27-51.

Piippo S, He X-L, Juslén A, Tan BC, Murphy DH \& Pócs T (2002) Hepatic and hornwort flora of Singapore. Annales Botanci Fennici 39: 101-128.

Piippo S, He X-L, Koponen T \& Redfearn P, Li J-X (1998) Hepaticae from Yunnan, China, with a checklist of Yunnan Hepaticae and Anthocerotae. Journal of the Hattori Botanical Laboratory 84: $135-158$.

Pócs T (1984) Synopsis of the African Lepidoziaceae K. Müll. Pp. 107-119 in Váňa J (ed.), Proceedings of the third meeting of the bryologists from Central and East Europe. (Univerzita Karlova: Praha).

Pócs T (2008a) Bryophytes from the Fiji Islands, III. The genus Phaeolejeunea Mizut. (Lejeuneaceae), with detailed description of P. amicorum (Hürl.) Pócs, stat. nov. Fieldiana, Botany 47: 139-145.

Pócs T (2008b) Bryophytes from the Fiji Islands, IV. The genus Frullania Raddi (Jungermanniopsida), I., with description of F. vivipara Pócs, spec. nov. Fieldiana, Botany 47: 147-158.

Pócs T \& Eggers J (2007) Bryophytes from the Fiji Islands, II. An acccount of the genus Colura, with a description of $C$. vitiensis sp. nov. Polish Botanical Journal 52: 81-92.

Pócs T, Mizutani M \& Piippo S (1994) Bryophyte Flora of the Huon Peninsula, Papua-NewGuinea LXV. Preliminary Contributions on Lejeuneaceae (Hepaticae).1. Annales Botanci Fennici 31: 179-190.

Pócs T \& Ninh T (2005) Contribution to the bryoflora of Vietnam. VI. On the liverwort flora of Vu Quang Nature Reserve. Acta Botanica Hungarica 47: 151-171.

Pócs T, Sass-Gyarmati A, Naikatini A, Braggins J, Pócs S. \& von Konrat M (2011) New liverwort (Marchantiophyta) records for the Fiji Islands. Telopea 13(3): 455-494.

Pócs T \& Streimann H (1999) Epiphyllous liverworts from Queensland, Australia. Bryobrothera 5: $165-172$.

Pócs T, Tixier P \& Jovet-Ast S (1967) Adatok Észak-Vietnam mohaflórájához. II. Seconde contribution à la bryoflore du Nord Vietnam. Botanikai közlemények 54: 27-38.

Renner MAM \& Brown EA (2008) Mnioloma (Calypogeiaceae: Jungermanniopsida) in Australasia: how many species are there?. Fieldiana, Botany 47: 159-174.

Renner MAM \& de Lange PJ (2009) Radula multiflora Gottsche ex Schiffn. - a new record for the Chatham Islands, New Zealand. Australasian Bryological Newsletter 57: 12-13. 
Sass-Gyarmati A (2003) Records on the distribution of Ptychanthoideae (subfam. of Lejeuneaceae, Hepaticae) in Australia. Tropical Bryology 24: 21-31.

Sass-Gyarmati A (2005) Lopholejeunea pocsii Gyarmati (Lejeuneaceae, subfam. Ptychanthoideae), a new species of subgenus Pholianthus B. Thiers \& Gradst. from the Fiji-Islands. Cryptogamie, Bryologie 26: 403-410.

Sass-Gyarmati A (2008) Bryophytes from the Fiji Islands, V. Lopholejeunea vojtkoana Gyarmati sp. nov. from Fiji Islands (Lejeuneaceae, subfam. Ptychanthoideae). Nova Hedwigia 87: 479-486.

Schiffner V (1890) Lebermoose (Hepaticae), mit Zugrundelegung der von Dr. A. C. M. Gottsche ausgeführten Vorarbeiten. Pp. 1-45 in Naumann FC (ed.) Die Forschungsreise S. M. S. "Gazelle" in der Jahren 1874 bis 1877 unter Kommando des Kapitän zur See Freiherrn von Schleinitz, Vol. IV. (Berlin).

Schiffner V (1898) Conspectus Hepaticarum Archipelagi Indici. (Batavia).

Schiffner V (1900) Die Hepaticae der Flora von Buitenzorg. I. Band. Enthaltend die Beschreibung aller bisher aus Java bekannt gewordenen Ricciaceae, Marchantiaceae, Jungermaniaceae Anakrogynae und Jungermaniaceae Akrogynae Unterfam. Epigoniantheae. (E. J. Brill: Leiden).

Schiffner V (1955) Die Lebermoose der Deutschen Limnologischen Sunda-Expedition. Archiv für Hydrobiologie, supplement 21: 382-407.

Schuster RM (1963) Studies in antipodal Hepaticae I. Annotated key to the genera of antipodal Hepaticae with special reference to New Zealand and Tasmania. Journal of the Hattori Botanical Laboratory 26: 185-309.

Schuster RM (1964) Studies on antipodal Hepaticae VI. The suborder Perssonellinae: morphology, anatomy and possible evolution. Bulletin of the Torrey Botanical Club 91: 479490.

Schuster RM (1968) Studies on the Hepaticae, XXIX-XLIV. A miscellany of new taxa and new range extensions. Nova Hedwigia 15: 437-529.

Schuster RM (1969) Problems of the antipodal distribution in lower land plants. Taxon 18: 46-91.

Schuster RM (1970) Studies on Antipodal Hepaticae, III. Jubulopsis Schuster, Neohattoria Kamimura and Amphijubula Schuster. Journal of the Hattori Botanical Laboratory 33: 266-304.

Schuster RM (1980a) Studies on Hepaticae. LIV-LVIII. Kurzia v. Mart. (Microlepidozia (Spr.) Joerg.), Megalembidium Schust., Psiloclada Mitt., Drucella Hodgs. and Isolembidium Schust. Journal of the Hattori Botanical Laboratory 48: 337-421.

Schuster RM (1980b) The Hepaticae and Anthocerotae of North America, vol. IV. (Columbia University Press: New York).

Schuster RM (1984) Phytogeography of the Bryophyta. Pp. 463-626 in Schuster RM (ed.), New Manual of Bryology, vol. 1. (Hattori Botanical Laboratory: Nichinan).

Schuster RM (1992a) The Hepaticae and Anthocerotae of North America, vol. V. (Columbia University Press: New York).

Schuster RM (1992b) The Hepaticae and Anthocerotae of North America, vol. VI. (Columbia University Press: New York).

Schuster RM (1999) Studies on Hepaticae LXVI. Lepidoziaceae subfam. Zoopsidoideae (3): Zoopsidella. Nova Hedwigia 69: 101-150.

Schuster RM (2000) Austral Hepaticae, Part. 1. Nova Hedwigia, Beiheft 118: 1-524.

Schuster RM (2002) Austral Hepaticae, part II. Nova Hedwigia, Beiheft 119: 1-606.

Schuster RM \& Konstantinova NA (1995) Studies on Treubiales, I. On Apotreubia Hatt. et al. and A. hortonae Schust. \& Konstantinova, sp. n. Journal of the Hattori Botanical Laboratory 78: 41-61.

Schuster RM \& Scott GAM (1969) A study of the family Treubiaceae (Hepaticae; Metzgeriales). Journal of the Hattori Botanical Laboratory 32: 219-257.

Scott GAM \& Bradshaw JA (1985) Australian liverworts (Hepaticae): Annotated list of binominals and check-list of published species with bibliography. Brunonia 8: 1-171. 
Singh AP, Kumar D \& Nath V (2008) Studies on the genera Frullania Raddi and Jubula Dum. from Meghalaya (India): Eastern Himalayas. Taiwania 53: 51-84.

So ML (2000) Studies on Plagiochila (Hepaticae) in Australasia and the Pacific II. Cryptogamie, Bryologie 21: 223-231.

So ML (2001a) Plagiochila (Hepaticae, Plagiochilaceae) in China. Systematic Botany Monographs 60: $1-214$.

So ML (2001b) Recognized species of Plagiochila in Australasia and some island groups of the Pacific Ocean. New Zealand Journal of Botany 39: 395-421.

So ML (2002) The Genus Porella (Porellaceae, Hepaticae) in Australasia and the South Pacific. Systematic Botany 27: 4-13

So ML (2006) Radula (Radulaceae, Marchantiophyta) in the South Pacific. Journal of the Hattori Botanical Laboratory 99: 207-232.

So ML \& Grolle R (1999) Studies on Plagiochila in Asia: supplements to sections Abietinae, Annotinae, Ciliatae, Contiguae, Cucullatae, Poeltiae, Subtropicae and Zonatae. Cryptogamie, Bryologie 20: 167-179.

So ML \& Grolle R (2000) Studies on Plagiochila (Hepaticae) in Australasia and the Pacific. I. Cryptogamie, Bryologie 21: 7-18.

So ML \& Grolle R (2001) On Plagiochila subgenus Plagiochila section Abietinae (Hepaticae). Systematic Botany 26: 459-469.

Söderström L, Gradstein SR \& Hagborg A (2010) Checklist of hornworts and liverworts for Java. Phytotaxa 9: 53-149.

Söderström L, Hagborg A, von Konrat M \& Renner MAM (2008) Early Land Plants Today: Liverwort checklist of checklists. Fieldiana, Botany, n.s. 47: 105-130.

Söderström L, Urmi E \& Váňa J (2007) The distribution of Hepaticae and Anthocerotae in Europe and Macaronesia - Update 1-427. Cryptogamie, Bryologie 28: 299-350.

Solari SS (1983) Patagonian bryophytes 8. On the identity of Lepicolea scolopendra (Hook.) Trev. var. magellanica Gola and Sendtnera ochroleuca (Spreng.) Nees var. piligera De Not. Lindbergia 9: 86-88.

Stephani F (1893a) Hepaticarum species novae. Pars I. Hedwigia 32: 17-29.

Stephani F (1893b) Hepaticarum species novae. Pars II. Hedwigia 32: 137-147.

Stephani F (1894) Hepaticarum species novae VI. Hedwigia 33: 137-169.

Stephani F (1896) Hepaticarum species novae IX. Hedwigia 35: 73-140.

Stephani F (1896) [1897] Hepaticae (in: Reinecke F, Die Flora der Samoa-Inseln). Botanische Jahrbücher für Systematik, Pflanzengesichte und Pflanzengeographie 23: 300-316.

Stephani F (1898) Species Hepaticarum 1. Bulletin de l'Herbier Boissier 6 ( 5): 361-378.

Stephani F (1899a) Species Hepaticarum 1. Bulletin de l'Herbier Boissier 7 (7): 518-533.

Stephani F (1899b) Species Hepaticarum 1. Bulletin de l'Herbier Boissier 7 (9): 655-695.

Stephani F (1899c) Species Hepaticarum 1. Bulletin de l'Herbier Boissier 7 (10): 727-764.

Stephani F (1899d) Species Hepaticarum 1. Bulletin de l'Herbier Boissier 7: 927-956.

Stephani F (1900) Species Hepaticarum 1. Mémoires de l'Herbier Boissier suite au Bulletin de l'Herbier Boissier 11: 1-49.

Stephani F (1901) Species Hepaticarum 2. Bulletin de l'Herbier Boissier, série 2 1: 1022-1151.

Stephani F (1903a) Species Hepaticarum 2. Bulletin de l'Herbier Boissier, série 2 3: 98-129.

Stephani F (1903b) Species Hepaticarum 2. Bulletin de l'Herbier Boissier, série 2 3: 522-537.

Stephani F (1903c) Species Hepaticarum 2. Bulletin de l'Herbier Boissier, série 2 3: 959-974.

Stephani F (1904a) Species Hepaticarum 2. Bulletin de l'Herbier Boissier, série 2 4: 18-32.

Stephani F (1904b) Species Hepaticarum 2. Bulletin de l'Herbier Boissier, série 2 4: 153-168.

Stephani F (1908a) Species Hepaticarum 3. Bulletin de l'Herbier Boissier, série 2 8(11): 837-866.

Stephani F (1908b) Species Hepaticarum 3. Bulletin de l'Herbier Boissier, série 2 8(12): 941-966.

Stephani F (1909) Species Hepaticarum 4. (George \& Co.: Genève \& Bale).

Stephani F (1910) Species Hepaticarum 4. (George \& Co.: Genève \& Bale).

Stephani F (1911) Species Hepaticarum 4. (George \& Co.: Genève \& Bale).

Stephani F (1912) Species Hepaticarum 5. (George \& Co.: Genève \& Bale). 
Stephani F (1913) Species Hepaticarum 5. (George \& Co.: Genève \& Bale).

Stephani F (1914) Species Hepaticarum 5. (George \& Co.: Genève \& Bale).

Stephani F (1915) Species Hepaticarum 5. (George \& Co.: Genève \& Bale).

Stephani F (1916) Species Hepaticarum 5. (George \& Co.: Genève \& Bale).

Stephani F (1922) Species Hepaticarum 6. (George \& Co.: Genève \& Bale).

Thiers BM (1983) Index to the genera and species of Hepaticae described by William Mitten. Brittonia 35: 271-300.

Thiers BM (1985) Branching in Lejeuneaceae III. Ptychanthoideae. Nova Hedwigia, Beiheft 80: 31-61.

Thiers BM (1992a) New species of Cheilolejeunea and Otolejeunea (Hepaticae, Lejeuneaceae) from Australia. Brittonia 44: 160-165.

Thiers BM (1992b) A re-evaluation of Cheilolejeunea subgenus Xenolejeunea. Tropical Bryology 5: $10-21$.

Thiers BM (1993) A monograph of Pleurozia (Hepaticae, Pleuroziaceae). The Bryologist 96: $517-554$.

Thiers BM \& Gradstein SR (1989) Lejeuneaceae (Hepaticae) of Australia. I. Subfamily Ptychanthoideae. Memoirs of the New York Botanical Garden 52: 1-79.

Tixier P (1970) Bryophytae Indosinicae. Bryophytes from Thai Tenasserim. Natural history bulletin of the Siam society 23: 541-559.

Tixier P (1985) Contribution à la connaissance des Cololejeunoideae. Bryophytorum Bibliotheca 27: $1-439$.

Udar R \& Awasthi US (1982) The genus Drepanolejeunea St. in India. Journal of the Hattori Botanical Laboratory 53: 419-437.

Váňa J (1973a) Lebermoose aus Neuguinea. 10. Jungermannia. Journal of the Hattori Botanical Laboratory 37: 185-190.

Váňa J (1973b “1972") Miscellaneous notes on the Asiatic Jungermannioideae II. Journal of the Hattori Botanical Laboratory 36: 57-74.

Váňa J (1975) Studien über die Jungermannioideae (Hepaticae). 8. Jungermannia subg. Plectocolea und Solenostoma in Australien, Neuseeland und Ozeanien. Folia Geobotanica et Phytotaxonomica 10: 277-323.

Váňa J (1991a) The bryophytes of Sabah (North Borneo) with special reference to the BRYOTROP transect of Mt. Kinabalu. XIII. Jungermanniaceae (Hepaticopsida, Jungermannialaes). Willdenowia 20: 171-183.

Váňa J (1991b) The bryophytes of Sabah (North Borneo) with special reference to the BRYOTROP transect of Mt. Kinabalu. XV. Lophoziaceae (Hepaticae). Willdenowia 20: 199-219.

Váňa J \& Inoue H (1983) Studies in Taiwan Hepaticae. V. Jungermanniaceae. Bulletin of the National Science Museum, Tokyo. Series B, Botany 9: 125-142.

Váňa J \& Piippo S (1989a) Bryophyte flora of the Huon Peninsula, Papua New Guinea. XXIX. Jungermanniaceae and Gymnomitraceae (Hepaticae). Annales Botanci Fennici 26: 107-125.

Váňa J \& Piippo S (1989b) Bryophyte flora of the Huon Peninsula, Papua New Guinea. XXXI. Cephaloziaceae subfam. Allobielloideae, Cephaloziellaceae, Antheliaceae and Lophoziaceae (Hepaticae). Annales Botanci Fennici 26: 263-290.

Váňa J, Piippo S \& Koponen T (2005) Bryophyte flora of Hunan Province, China, 6. Jungermanniaceae and Gymnomitraceae (Hepaticae). Acta Botanica Fennica 178: 57-78.

Verdoorn F (1928) Kritische bemerkungen ueber ostasiatische und ozeanische Frullania-Arten aus dem subgenus Homotropantha (De Frullaniaceis III). Revue Bryologique et Lichénologique 1: $109-122$.

Verdoorn F (1930) Revision der von Ozeanien angefuhrten Frullaniaceae (De Frullaniaceis VIII). Nederlandsch Kruidkundig Archief. Verslagen en Mededelingen der Nederlandsche Botanische Vereeniging 1930: 155-175.

Verdoorn F (1934) Die Frullaniaceis XIV. Revision der von Ozeanien, Australien und Neuseeland angeführten Lejeuneaceae Holostipae. Blumea 1: 216-240. 
Verdoorn F (1937) On some new collections of Asiatic and Oceanic Jubulae (De Frullaniaceis XVIII). Blumea Supplement 1: 210-213.

von Konrat M, Naikatini A, Tuiwawa M, Söderström L, Fife A, Renner M, Brownsey P, Perrie L, Hagborg A, Pócs T, Lumbsch HT, Braggins J, Séneca A \& Brown E (2011) A brief history of the cryptogams of Fiji and prospects for the future. Telopea 13:361-374.

von Konrat MJ, Braggins JE, Asakawa Y \& Toyota M (2006) Frullania chevalieri (Jubulaceae) in New Zealand, with a reassessment of Schusterella. The Bryologist 109: 141-156.

von Konrat M, Pócs T, Naikatini A, Tuiwawa M, Söderström L, Brownsey P, Braggins J, Brown E \& Fife A (2011) Fiji cryptogams: A brief history and prospects for the future. Telopea 13(3): 361-374.

von Konrat MJ, Söderström L, Hagborg A, Crosby MR \& Engel JJ (2010b) Early Land Plants Today: Index of liverworts \& hornworts 2006-2008. Cryptogamie, Bryologie 31: 3-30.

von Konrat M, Söderström L \& Hagborg A (2010a) The Early Land Plants Today project: A community-driven effort and a new partnership with Phytotaxa. Phytotaxa 9: 11-21.

Wilson R, Gradstein SR, Schneider H \& Heinrichs J (2007) Unravelling the phylogeny of Lejeuneaceae (Jungermanniopsida): evidence for four lineages. Molecular Phylogenetics and Evolution 43: 270-282.

Yamada K (1975) Radula collections made by Dr. H. Inoue in Ceylon. Journal of Japanese Botany [Shokubutsu kenkyu zasshi] 50: 372-378.

Yamada K (1979) A revision of Asian taxa of Radula, Hepaticae. Journal of the Hattori Botanical Laboratory 45: 201-322.

Yamada K (1984) Notes on new record of three Radula species (Hepaticae) from Fiji. Proceedings of the bryological society of Japan 3: 181-182.

Yamada K (1985) Radula collections made by Drs. Z. Iwatsuki and N. Kitagawa in New Caledonia. Journal of the Hattori Botanical Laboratory 58: 111-130.

Yamada K (1999) Taxonomy of the section Amentulosae of genus Radula (Radulaceae, Hepaticae). Haussknechtia, Beiheft 9: 391-398.

Yamada K \& Hayashi M (2003) Studies on the bryophyte flora of Vanuatu. 8. Heteroscyphus (Geocalycaceae, Hepaticae). Bulletin of the National Science Museum, Tokyo. Series B, Botany 29: 149-152.

Yamada K \& Piippo S (1989) Bryophyte flora of the Huon Peninsula, Papua New Guinea. XXXII. Radula (Radulaceae, Hepaticae). Annales Botanci Fennici 26: 349-387.

Yang J-D \& Lin S-H (2008) Cheilolejeunea falsinervis (Marchantiophyta) newly recorded for Taiwan. Bryological Research [Sentairui Kenkyu] 9: 319-321.

Yang J-D \& Lin S-H (2009) Lejeunea sordida (Marchantiophyta) newly recorded for Taiwan. Bryological Research [Sentairui Kenkyu] 9: 388-390.

Yuzawa Y (1991) A monograph of subgen. Chonanthelia of gen. Frullania (Hepaticae) of the world. Journal of the Hattori Botanical Laboratory 70: 181-291.

Zhu R-L (2006) On Cheilolejeunea tenella (Jungermanniopsida: Lejeuneaceae), a poorly known species from Singapore. Annales Botanci Fennici 43: 484-487.

Zhu R-L \& Gradstein SR (2005) Monograph of Lopholejeunea (Spruce) Schiffn. (Lejeuneaceae, Hepaticae) in Asia. Monographs in Systematic Botany from the Missouri Botanical Garden 74: 1-98.

Zhu R-L \& Grolle R (2004) Nomenclatural notes on Cheilolejeunea inaequitexta and C. trapezia (Lejeuneaceae, Hepaticae). Annales Botanci Fennici 41: 445-447.

Zhu R-L \& Lai M-J (2005) Cheilolejeunea decursiva (Sande Lac.) R. M. Schust. and C. ventricosa (Schiffn.) X. L. He (Lejeuneaceae, Hepaticae) new to Australia. Journal of the Hattori Botanical Laboratory 98: 193-203.

Zhu R-L \& So ML (2001) Epiphyllous liverworts of China. Nova Hedwigia, Beiheft 121: 1-418. 